\title{
UNIQUENESS RESULTS FOR MATRIX-VALUED SCHRÖDINGER, JACOBI, AND DIRAC-TYPE OPERATORS
}

\author{
FRITZ GESZTESY, ALEXANDER KISELEV, AND KONSTANTIN A. MAKAROV
}

\begin{abstract}
Let $g(z, x)$ denote the diagonal Green's matrix of a self-adjoint $m \times m$ matrix-valued Schrödinger operator $H=-\frac{d^{2}}{d x^{2}} I_{m}+Q(x)$ in $L^{2}(\mathbb{R})^{m}$, $m \in \mathbb{N}$. One of the principal results proven in this paper states that for a fixed $x_{0} \in \mathbb{R}$ and all $z \in \mathbb{C}_{+}, g\left(z, x_{0}\right)$ and $g^{\prime}\left(z, x_{0}\right)$ uniquely determine the matrixvalued $m \times m$ potential $Q(x)$ for a.e. $x \in \mathbb{R}$. We also prove the following local version of this result. Let $g_{j}(z, x), j=1,2$ be the diagonal Green's matrices of the self-adjoint Schrödinger operators $H_{j}=-\frac{d^{2}}{d x^{2}} I_{m}+Q_{j}(x)$ in $L^{2}(\mathbb{R})^{m}$. Suppose that for fixed $a>0$ and $x_{0} \in \mathbb{R},\left\|g_{1}\left(z, x_{0}\right)-g_{2}\left(z, x_{0}\right)\right\|_{\mathbb{C}^{m \times m}}+$ $\left\|g_{1}^{\prime}\left(z, x_{0}\right)-g_{2}^{\prime}\left(z, x_{0}\right)\right\|_{\mathbb{C}^{m \times m}} \underset{|z| \rightarrow \infty}{=} O\left(e^{-2 \operatorname{Im}\left(z^{1 / 2}\right) a}\right)$ for $z$ inside a cone along the imaginary axis with vertex zero and opening angle less than $\pi / 2$, excluding the real axis. Then $Q_{1}(x)=Q_{2}(x)$ for a.e. $x \in\left[x_{0}-a, x_{0}+a\right]$.

Analogous results are proved for matrix-valued Jacobi and Dirac-type operators.
\end{abstract}

This is a revised and updated version of a previously archived file.

\section{INTRODUCTION}

While various aspects of inverse spectral theory for scalar Schrödinger, Jacobi, and Dirac-type operators, and more generally, for $2 \times 2$ Hamiltonian systems, are well-understood by now, the corresponding theory for such operators and Hamiltonian systems with $m \times m, m \in \mathbb{N}$, matrix-valued coefficients is still in its infancy. A particular inverse spectral theory aspect we have in mind is that of determining isospectral sets (manifolds) of such systems. It may, perhaps, come as a surprise that determining the isospectral set of Hamiltonian systems with matrix-valued periodic coefficients is a completely open problem. It appears to be no exaggeration to claim that (unless one considers trivial cases such as diagonal coefficient matrices, etc.) absolutely nothing seems to be known about the corresponding isospectral sets of periodic matrix-valued Schrödinger operators with the sole exception of its compactness. The same ignorance applies to Jacobi, Dirac, and more generally, to periodic $2 m \times 2 m$ Hamiltonian systems with $m \geq 2$. While the present paper is not sufficiently ambitious to change this sorry state of affairs, we will take a modest step toward a closer investigation of inverse spectral problems and prove a few uniqueness theorems for such systems, that is, determine spectral data (interpreted in a very broad sense) that uniquely determine the matrix-valued coefficients in Schrödinger, Jacobi, and Dirac-type systems. It should be mentioned that these

1991 Mathematics Subject Classification. Primary 34E05, 34B20, 34L40; Secondary 34A55.

Key words and phrases. Uniqueness results, Schrödinger, Dirac, and Jacobi operators, WeylTitchmarsh matrices, Green's matrices.

To appear in Math. Nachr. 
types of problems are not just of interest in a spectral theoretic context, but due to their implications for other areas such as completely integrable systems (e.g., the nonabelian Korteweg-deVries, Toda lattice, and Ablowitz-Kaup-Newell-Segur (nonlinear Schrödinger) hierarchies), are also of considerable interest to a much larger audience.

Before we continue along this line of thought, it seems appropriate to briefly mention some of the present day knowledge of $2 m \times 2 m$ matrix-valued Hamiltonian systems and the Weyl-Titchmarsh and spectral theory associated with them. To save space we will often simultaneously discuss references on Schrödinger, Dirac, and general Hamiltonian systems together without differentiating between them, and occasionally single out the finite-difference (Jacobi) systems. Moreover, there exists a considerable amount of literature on the foundations of Hamiltonian systems (and their special cases, such as Schrödinger, Jacobi, and Dirac-type systems) which necessarily forces us to be rather selective here. In particular, we focus primarily on the case $m \geq 2$ for the remainder of this introduction.

The basic Weyl-Titchmarsh theory of regular Hamiltonian systems can be found in Atkinson's monograph [12]; Weyl-Titchmarsh theory of singular Hamiltonian systems and their basic spectral theory was developed by Hinton and Shaw and many others (see, e.g., [2], 65]- 67], [77], 78], [83, 84], [93], 113], 116], 118], [122, [128], 132, Ch. 9], [140] and the references therein); the corresponding theory for Jacobi systems can be found in [19], [48], 130] and the literature therein. Various aspects of direct spectral theory, including investigations of the nature of the spectrum involved, (regularized) trace formulas, etc., appeared in [15], 25], [27], [28, [33], 35], [38], 39], [55], 64], [86], [89], [109], 114], 119]. General asymptotic expansions of Weyl-Titchmarsh matrices as the (complex) spectral parameter tends to infinity under optimal regularity assumptions on the coefficients are of relatively recent origin and can be found in [34], [36], 337] (see also [126], 137]). The inverse scattering formalism for continuous Hamiltonian systems has been studied by a variety of authors and we refer, for instance, to [1], [1], [5], [10], [11], [49], [108], [110], [11], 138], 145. General inverse spectral theory, the existence of transformation operators, etc., is discussed in [62, [92], 99], 101], 121], 124, 125], 128], 1129, 131, [132], and the references therein. Inverse monodromy problems for canonical systems received a lot of attention recently. The interested reader is referred to [7, 8], 9], 100]-102], 127, 132] and the extensive literature cited therein. The corresponding inverse spectral and scattering theory for matrix-valued finite difference systems and its intimate connection to matrix-valued orthogonal polynomials is treated in [3], [6], [19], [40], [46]- 48], [53], [54], 97], [98], 130]. More specific inverse spectral problems, such as compactness of the isospectral set of periodic Schrödinger operators [26], special isospectral matrix-valued Schrödinger operators, and Borg-type uniqueness theorems (for periodic coefficients as well as eigenvalue problems on compact intervals) were recently studied in [31], 32], 37], [38, 42], 73], [74, [99], 101], 102], 132], 133]. Moreover, direct spectral theory in the particular case of periodic Hamiltonian systems (i.e., Floquet theory and alike) has been studied in [26], 27], [38], [41], 442], [52], [75], [85], [86], 117], 142-114, with many more pertinent references to be found therein.

Apart from Floquet theoretic applications in connection with Hamiltonian systems already briefly touched upon, we also need to mention applications to random Schrödinger operators associated with strips as discussed, for instance, in [29], [82], 87, [88], and especially to nonabelian completely integrable systems. Since the 
literature associated with the latter topic is of enormous proportions, we can only refer to a few pertinent publications, such as, [11], [20], [21], 30], [43]- 45], [51], [103, 107, 108, 112, 123, [126-128], 130. The interested reader will find a wealth of additional material in these references.

Finally, we turn to the principal subject of this paper, that is, uniqueness-type theorems for Schrödinger, Jacobi, and Dirac-type operators. We have already mentioned a few uniqueness results in connection with Borg-type theorems for such systems. Additional uniqueness-type results in terms of matrix-valued Wronskians and transformation operators can be found in some papers by Leibenzon [90], [91] and Malamud [100], 101]. The uniqueness theorems proven in this paper are directly formulated in terms of diagonal Green's matrices $g\left(z, x_{0}\right)$ and their $x$-derivatives $g^{\prime}\left(z, x_{0}\right)$ at some fixed $x_{0} \in \mathbb{R}$, for matrix-valued Schrödinger and Dirac-type operators on $\mathbb{R}$ and similarly in terms of diagonal Green's matrices $g\left(z, k_{0}, k_{0}\right)$ and off-diagonal Green's matrices $G\left(z, k_{0}, k_{0}+1\right)$, etc., for some fixed $k_{0} \in \mathbb{Z}$, in the context of matrix-valued Jacobi operators on $\mathbb{Z}$. Moreover, we prove certain localized versions of these uniqueness theorems for exponentially close diagonal Green's matrices with respect to $z$ as $|z| \rightarrow \infty$ in the Schrödinger and Dirac-type context and analogous theorems in the case of Jacobi operators whose (diagonal and certain off-diagonal) Green's matrices differ by an inverse power of $z$ as $|z| \rightarrow \infty$. To be a bit more specific, we briefly describe some of our principal results in the case of self-adjoint Schrödinger operators $H$ in $L^{2}(\mathbb{R})^{m}, m \in \mathbb{N}$ associated with $m \times m$ matrix-valued differential expressions $-\frac{d^{2}}{d x^{2}} I_{m}+Q(x)$, with $Q=Q^{*} \in L_{\text {loc }}^{1}(\mathbb{R})^{m \times m}$.

Let $\mathbb{C}_{+}$be the open complex upper half-plane. Denoting by $g(z, x), z \in \mathbb{C} \backslash \mathbb{R}$, $x \in \mathbb{R}$ the diagonal Green's matrix associated with $H$ (i.e., the integral kernel of the resolvent of $H$ on the diagonal, $\left.g(z, x)=(H-z)^{-1}(x, x)\right)$ and by $g^{\prime}(z, x)$ its $x$-derivative, we will prove the following result (cf. Theorem 3.7).

Theorem 1.1. Fix $x_{0} \in \mathbb{R}$. Then $g\left(z, x_{0}\right)$ and $g^{\prime}\left(z, x_{0}\right)$ for all $z \in \mathbb{C}_{+}$uniquely determine the matrix-valued $m \times m$ potential $Q(x)$ for a.e. $x \in \mathbb{R}$.

Moreover, let $H_{j}, j=1,2$ be two Schrödinger operators associated with the $m \times m$ matrix-valued potentials $Q_{j}(x)$ and $g_{j}(z, x)$ the corresponding diagonal Green's matrices of $H_{j}$. Then we prove the following local version of Theorem 1.1 (cf. Theorem 3.8).

Theorem 1.2. Let $a>0$ and $x_{0} \in \mathbb{R}$. If

$$
\begin{aligned}
& \left\|g_{1}\left(z, x_{0}\right)-g_{2}\left(z, x_{0}\right)\right\|_{\mathbb{C}^{m \times m}}+\left\|g_{1}^{\prime}\left(z, x_{0}\right)-g_{2}^{\prime}\left(z, x_{0}\right)\right\|_{\mathbb{C}^{m \times m}} \\
& \underset{|z| \rightarrow \infty}{=} O\left(e^{-2 \operatorname{Im}\left(z^{1 / 2}\right) a}\right) \\
& \text { with } 0<\arg (z)<\pi \text {, then } \\
& \qquad Q_{1}(x)=Q_{2}(x) \text { for a.e. } x \in\left[x_{0}-a, x_{0}+a\right] .
\end{aligned}
$$

along a ray with $0<\arg (z)<\pi$, then

Our results were of course inspired by analogous ones in the special scalar context $m=1$, but also by a very interesting uniqueness theorem proven by Berezanskii in 1953 [17, [18 in the context of multi-dimensional Schrödinger operators $H$. Berezanskii's point of departure is a bit different from ours. He considered the spectral kernel $\vartheta(\lambda, x, y)$ of $H$ (i.e., the integral kernel of the spectral projection $E_{H}(\lambda)$ of $\left.H, \vartheta(\lambda, x, y)=E_{H}(\lambda, x, y)\right)$ and appropriate normal derivatives of it with respect to $x$ and $y$ across an arbitrarily small piece of a smooth surface in $\mathbb{R}^{d}$, $d=2,3$. There are additional hypotheses in Berezanskii's work which need not 
be discussed here. In a certain sense we replaced the spectral projection of $H$ by its resolvent and (in our one-dimensional context) the arbitrarily small piece of a surface by the point $x_{0}$ and the corresponding normal derivative by $d / d x$.

In Section 2 we review the basic Weyl-Titchmarsh theory for matrix-valued Schrödinger and Dirac-type operators as needed in our principal Section 3. The latter contains our uniqueness results for Schrödinger and Dirac-type operators. The final Section 1 then treats Weyl-Titchmarsh theory and uniqueness theorems for matrix-valued Jacobi operators.

\section{Matrix-Valued Schrödinger and Dirac-Type Operators}

In this section we briefly recall the Weyl-Titchmarsh theory for matrix-valued Schrödinger and Dirac-type operators. In order to treat both cases in parallel, we use the fact that both are special cases of Hamiltonian systems and hence develop the theory from that point of view. Throughout this paper all matrices will be considered over the field of complex numbers $\mathbb{C}$ and the corresponding linear space of $k \times \ell$ matrices will be denoted by $\mathbb{C}^{k \times \ell}$.

The basic assumption for Sections 2 and 3 of this paper will be the following.

Hypothesis 2.1. Fix $m \in \mathbb{N}$ and define the $2 m \times 2 m$ matrix

$$
J=\left(\begin{array}{cc}
0 & -I_{m} \\
I_{m} & 0
\end{array}\right)
$$

We consider two principal cases: Either

(i) suppose $Q=Q^{*} \in L_{\mathrm{loc}}^{1}(\mathbb{R})^{m \times m}$ and introduce the $2 m \times 2 m$ matrices

$$
A=\left(\begin{array}{cc}
I_{m} & 0 \\
0 & 0
\end{array}\right), \quad B(x)=\left(\begin{array}{cc}
-Q(x) & 0 \\
0 & I_{m}
\end{array}\right)
$$

or

(ii) suppose

$$
A=I_{2 m}, \quad B=B^{*} \in L_{\mathrm{loc}}^{1}(\mathbb{R})^{2 m \times 2 m} .
$$

Given Hypothesis 2.1 we consider the Hamiltonian system

$$
J \Psi^{\prime}(z, x)=(z A+B(x)) \Psi(z, x) \text { for a.e. } x \in \mathbb{R},
$$

where $z \in \mathbb{C}$ plays the role of a spectral parameter and $\Psi(z, x)$ is assumed to satisfy

$$
\Psi(z, \cdot) \in \mathrm{AC}_{\text {loc }}(\mathbb{R})^{2 m \times 2 m} .
$$

Here and later on, $I_{p}$ denotes the identity matrix in $\mathbb{C}^{p \times p}$ for $p \in \mathbb{N}, M^{*}$ the adjoint (i.e., complex conjugate transpose), $M^{t}$ the transpose of the matrix $M$, and $\mathrm{AC}_{\text {loc }}(\mathbb{R})$ denotes the set of locally absolutely continuous functions on $\mathbb{R}$. At times it will be convenient to consider a $2 m \times r$ solution matrix of (2.4), with $r=1, \ldots, 2 m$, whose entries will then be assumed to lie in $\mathrm{AC}_{\text {loc }}(\mathbb{R})$.

One verifies that Hypothesis 2.1(i) governs the case of matrix-valued Schrödinger operators. In fact, inserting

$$
\Psi(z, x)=\left(\begin{array}{ll}
\psi_{1}(z, x) & 0 \\
\psi_{2}(z, x) & 0
\end{array}\right)
$$

into equation 2.4 then yields

$$
\begin{aligned}
-\psi_{1}^{\prime \prime}(z, x)+Q(x) \psi_{1}(z, x) & =z \psi_{1}(z, x), \\
\psi_{2}(z, x) & =\psi_{1}^{\prime}(z, x) .
\end{aligned}
$$


Here it is assumed that

$$
\psi_{j}(z, \cdot) \in \mathrm{AC}_{\mathrm{loc}}(\mathbb{R})^{m \times m}, \quad j=1,2 .
$$

In the case of Hypothesis 2.1(ii), (2.4) represents a Dirac-type system.

In connection with our uniqueness result for Dirac-type operators in Section 3 we will make the following additional assumption due to the freedom of certain gauge transformations in the Dirac context, which leaves the corresponding spectral matrix, but not the potential $B(x)$ invariant, as discussed by Gasymov 49. and Gasymov and Levitan [50].

Hypothesis 2.2. Suppose Hypothesis 2.1 and in the case of Dirac-type operators (i.e., assuming (2.3)) suppose that $B$ is locally essentially bounded, $B \in$ $L_{\mathrm{loc}}^{\infty}(\mathbb{R})^{2 m \times 2 m}$, and that $B(x)$ is of the special form

$$
\begin{aligned}
& B(x)=\left(\begin{array}{cc}
B_{1,1}(x) & B_{1,2}(x) \\
B_{1,2}(x) & -B_{1,1}(x)
\end{array}\right), \\
& \text { with } B_{1,1}(x)=B_{1,1}(x)^{*}, B_{1,2}(x)=B_{1,2}(x)^{*}, j=1,2 .
\end{aligned}
$$

Equation (2.10) represents a typical normal form inspired by the requirement

$$
J B(x)+B(x) J=0 \text { for a.e. } x \in \mathbb{R} .
$$

Various possible normal forms for Dirac-type operators are discussed in [50], [92], [99, [101], [109], and in the monographs [94, Ch. 9], [96, Ch. 7], 106, p. 193195]. The continuity assumption on $B$ in Hypothesis 2.2 is further discussed in the paragraph following Theorem 2.12.

Next we briefly turn to Weyl-Titchmarsh theory associated with (2.4) and recall some of the results developed by Hinton and Shaw in a series of papers devoted to spectral theory of (singular) Hamiltonian systems [68]- 72] (see also [83, 84]). While they discuss (2.4) under much more general hypotheses on $A(x)$ and $B(x)$, we confine ourselves here to the special cases of matrix-valued Schrödinger and Diractype systems governed by Hypothesis 2.1. The following facts on Weyl-Titchmarsh theory are taken from [36] and [37] and hence we omit the corresponding proofs.

Let $\Psi\left(z, x, x_{0}\right)$ be a normalized fundamental system of solutions of (2.4) at some $x_{0} \in \mathbb{R}$, that is, $\Psi\left(z, x, x_{0}\right)$ satisfies

$$
J \Psi^{\prime}(z, x)=(z A+B(x)) \Psi(z, x), \quad z \in \mathbb{C}
$$

for a.e. $x \in \mathbb{R}$, and

$$
\Psi\left(z, x_{0}, x_{0}\right)=I_{2 m}
$$

Moreover, we partition $\Psi\left(z, x, x_{0}\right)$ as

$$
\begin{aligned}
\Psi\left(z, x, x_{0}\right) & =\left(\psi_{j, k}\left(z, x, x_{0}\right)\right)_{1 \leq j, k \leq 2} \\
& =\left(\begin{array}{lll}
\Theta\left(z, x, x_{0}\right) & \Phi\left(z, x, x_{0}\right)
\end{array}\right)=\left(\begin{array}{cc}
\theta_{1}\left(z, x, x_{0}\right) & \phi_{1}\left(z, x, x_{0}\right) \\
\theta_{2}\left(z, x, x_{0}\right) & \phi_{2}\left(z, x, x_{0}\right)
\end{array}\right),
\end{aligned}
$$

where $\theta_{j}\left(z, x, x_{0}\right)$ and $\phi_{j}\left(z, x, x_{0}\right)$ for $j=1,2$ are $m \times m$ matrices, entire with respect to $z \in \mathbb{C}$, and normalized according to (2.13), that is,

$$
\theta_{1}\left(z, x_{0}, x_{0}\right)=\phi_{2}\left(z, x_{0}, x_{0}\right)=I_{m}, \quad \phi_{1}\left(z, x_{0}, x_{0}\right)=\theta_{2}\left(z, x_{0}, x_{0}\right)=0 .
$$

Next, let $\beta_{j} \in \mathbb{C}^{m \times m}, j=1,2$, introduce the matrix $\beta=\left(\beta_{1} \beta_{2}\right) \in \mathbb{C}^{2 m \times m}$, and assume that

$$
\operatorname{rank}(\beta)=m, \quad \beta \beta^{*}=I_{m}, \text { and either } \operatorname{Im}\left(\beta_{2} \beta_{1}^{*}\right) \leq 0 \text { or } \operatorname{Im}\left(\beta_{2} \beta_{1}^{*}\right) \geq 0 .
$$


One can then prove the following result.

Lemma 2.3 (see, e.g., 37]). Assume Hypothesis 2.1, let $\Theta\left(z, x, x_{0}\right)$ and $\Phi\left(z, x, x_{0}\right)$ be defined as in (2.14), and suppose $\beta$ satisfies (2.16). Then, for $c \in \mathbb{R} \backslash\left\{x_{0}\right\}$, $\beta \Phi\left(z, c, x_{0}\right)$ is singular if and only if $z$ is an eigenvalue for the regular boundary value problem given by (2.4), (2.5) together with the separated boundary conditions

$$
\left(I_{m} 0\right) \Psi\left(z, x_{0}\right)=0, \quad \beta \Psi(z, c)=0 .
$$

For the regular boundary value problem described in Lemma 2.3, the boundary conditions in (2.17) are self-adjoint whenever $\operatorname{Im}\left(\beta_{2} \beta_{1}^{*}\right)=0$.

Lemma 2.3 provides appropriate conditions for defining a certain meromorphic $m \times m$ matrix $M\left(z, c, x_{0}, \beta\right)$.

Definition 2.4. Assume Hypothesis 2.1 and let $\Theta\left(z, x, x_{0}\right)$, and $\Phi\left(z, x, x_{0}\right)$ be defined as in (2.14) with $\beta$ satisfying (2.16). For $c \neq x_{0}$, and $\beta \Phi\left(z, c, x_{0}\right)$ nonsingular let

$$
M\left(z, c, x_{0}, \beta\right)=-\left[\beta \Phi\left(z, c, x_{0}\right)\right]^{-1}\left[\beta \Theta\left(z, c, x_{0}\right)\right] .
$$

$M\left(z, c, x_{0}, \beta\right)$ is said to be the Weyl-Titchmarsh $M$-function for the regular boundary value problem described in Lemma 2.3.

The Weyl-Titchmarsh $M$-function in (2.18) is an $m \times m$ matrix-valued function with meromorphic entries whose poles correspond to eigenvalues for the regular boundary value problem given by (2.4), (2.5), and (2.17). Moreover, if $M \in \mathbb{C}^{m \times m}$, and one defines

$$
U\left(z, x, x_{0}\right)=\left(\begin{array}{l}
u_{1}\left(z, x, x_{0}\right) \\
u_{2}\left(z, x, x_{0}\right)
\end{array}\right)=\Psi\left(z, x, x_{0}\right)\left(\begin{array}{c}
I_{m} \\
M
\end{array}\right),
$$

with $u_{j}\left(z, x, x_{0}\right) \in \mathbb{C}^{m \times m}, j=1,2$, then $U\left(z, x, x_{0}\right)$ will satisfy the boundary condition at $x=c$ in (2.17) whenever $M=M\left(z, c, x_{0}, \beta\right)$. Intimately connected with the matrices introduced in Definition 2.4 is the set of $m \times m$ complex matrices known as the Weyl disk.

To describe this set, we first introduce the matrix-valued function $E(M)$ : With $c \neq x_{0}, z \in \mathbb{C} \backslash \mathbb{R}$, and with $U\left(z, c, x_{0}\right)$ defined by (2.12) in terms of a matrix $M \in \mathbb{C}^{m \times m}$, let

$$
E(M)=\sigma\left(x_{0}, c, z\right) U\left(z, c, x_{0}\right)^{*}(i J) U\left(z, c, x_{0}\right)
$$

where

$$
\sigma(s, t, z)=\frac{(s-t) \operatorname{Im}(z)}{|(s-t) \operatorname{Im}(z)|}, \quad \sigma(s, t)=\sigma(s, t, i), \quad \sigma(z)=\sigma(1,0, z),
$$

with $s, t \in \mathbb{R}, s \neq t$.

Definition 2.5. Assume Hypothesis 2.1 and fix $x_{0} \in \mathbb{R}, c \in \mathbb{R} \backslash\left\{x_{0}\right\}$, and $z \in \mathbb{C} \backslash \mathbb{R}$. Then $D\left(z, c, x_{0}\right)$ will denote the collection of all $M \in \mathbb{C}^{m \times m}$ for which $E(M) \leq 0$, where $E(M)$ is defined in (2.18). $D\left(z, c, x_{0}\right)$ is said to be a Weyl disk. The set of $M \in \mathbb{C}^{m \times m}$ for which $E(M)=0$ is said to be a Weyl circle (even when $m>1$ ).

This definition leads to a presentation that is a generalization of the description first given by Weyl [141]; a presentation which is geometric in nature, involves the contractive matrices $V \in \mathbb{C}^{m \times m}$, such that $V V^{*} \leq I_{m}$, and provides the justification for the geometric terms of circle and disk and (see, e.g., 68, 66, [83, $113)$. 
The disk has also been characterized in terms of matrices which statisfy hypothesis (2.16) and which serve as boundary data for the regular boundary value problem described in Lemma 2.3 (see, e.g. [13], [14). In particular, $\mathcal{D}\left(z, c, x_{0}\right)$ denotes the collection of all $M \in \mathbb{C}^{m \times m}$ obtained by the construction given in (2.18) where $c \neq x_{0}, z \in \mathbb{C} / \mathbb{R}$, where $\beta$ is the $m \times m$ matrices defined in hypothesis $(2.16)$ for which $\sigma\left(c, x_{0}, z\right) \operatorname{Im}\left(\beta_{2} \beta_{1}^{*}\right) \geq 0$.

We note that the Weyl circle corresponds to the regular boundary value problems in Lemma 2.3 with separated, self-adjoint boundary conditions.

Lemma 2.6 (71], 66], [83). Let $M \in \mathbb{C}^{m \times m}, c \neq x_{0}$, and $z \in \mathbb{C} \backslash \mathbb{R}$. Then, $E(M)=0$ if and only if there is a $\beta \in \mathbb{C}^{m \times 2 m}$ satisfying $\beta J \beta^{*}=0$ and $\beta \beta^{*}=I_{m}$ such that

$$
0=\beta U\left(z, c, x_{0}\right)
$$

where $U\left(z, c, x_{0}\right)$ is defined in (2.12) in terms of $M$. With $\beta$ so defined,

$$
M=-\left[\beta \Phi\left(z, c, x_{0}\right)\right]^{-1}\left[\beta \Theta\left(z, c, x_{0}\right)\right],
$$

that is, $M=M\left(z, c, x_{0}, \beta\right)$.

Next, we recall a fundamental property associated with matrices in $\mathcal{D}\left(z, c, x_{0}\right)$.

Lemma 2.7 (see, e.g., 37). If $M \in \mathcal{D}\left(z, c, x_{0}\right)$, then

$$
\sigma\left(c, x_{0}, z\right) \operatorname{Im}(M)>0 \text {. }
$$

Moreover, whenever $\beta \in \mathbb{C}^{m \times 2 m}$ satisfies $\beta J \beta^{*}=0$ and $\beta \beta^{*}=I_{m}$,

$$
M\left(\bar{z}, c, x_{0}, \beta\right)=M\left(z, c, x_{0}, \beta\right)^{*} .
$$

For $c>x_{0}$, the function $M\left(z, c, x_{0}, \beta\right)$, defined by (2.18), and satisfying (2.24), is said to be a matrix-valued Herglotz function of $\operatorname{rank} m$. Hence, for $\operatorname{Im}\left(\beta_{2} \beta_{1}^{*}\right)=$ 0 , poles of $M\left(z, c, x_{0}, \beta\right), c>x_{0}$, are at most of first order, are real, and have nonpositive residues. Such functions admit representations of the form

$$
M\left(z, c, x_{0}, \beta\right)=C_{1}+z C_{2}+\int_{-\infty}^{\infty} d \Omega\left(\lambda, c, x_{0}, \beta\right)\left(\frac{1}{\lambda-z}-\frac{\lambda}{1+\lambda^{2}}\right), \quad c>x_{0},
$$

where (with $\beta$ fixed) $C_{2} \geq 0$ and $C_{1}$ are constant $m \times m$ self-adjoint matrices, and $\Omega\left(\lambda, c, x_{0}, \beta\right)$ is a nondecreasing $m \times m$ matrix-valued function such that

$$
\begin{aligned}
& \int_{-\infty}^{\infty}\left\|d \Omega\left(\lambda, c, x_{0}, \beta\right)\right\|_{\mathbb{C}^{m \times m}}\left(1+\lambda^{2}\right)^{-1}<\infty \\
& \Omega\left((\lambda, \mu], c, x_{0}, \beta\right)=\lim _{\delta \downarrow 0} \lim _{\epsilon \downarrow 0} \frac{1}{\pi} \int_{\lambda+\delta}^{\mu+\delta} d \nu \sigma\left(c, x_{0}, \nu+i \epsilon\right) \operatorname{Im}\left(M\left(\nu+i \epsilon, c, x_{0}, \beta\right)\right) .
\end{aligned}
$$

For the self-adjoint boundary value problems in connection with Schrödinger, Dirac, and Jacobi operators discussed in this paper, $C_{2}=0$ in (2.26) (and also later in (2.41) and (2.55) ) and $\Omega\left(\lambda, c, x_{0}, \beta\right)$ is then piecewise constant with jump discontinuities at the eigenvalues of the problem. Analogous statements apply to $-M\left(z, c, x_{0}, \beta\right)$ if $c<x_{0}$. 
We further note that the sets $D\left(z, c, x_{0}\right)$ are closed, convex, and are nested with respect to increasing or decreasing values of $c$ (cf. [68], [70], [71], [83], [113]), that is,

$$
D\left(z, c_{2}, x_{0}\right) \subseteq D\left(z, c_{1}, x_{0}\right) \quad \text { for } \quad x_{0}<c_{1} \leq c_{2} \quad \text { or } \quad c_{2} \leq c_{1}<x_{0} .
$$

Hence, the intersection of this nested sequence, as $c \rightarrow \pm \infty$, is nonempty, closed and convex. We say that this intersection is a limiting set for the nested sequence.

Definition 2.8. Assume Hypothesis 2.1 and let $D_{ \pm}\left(z, x_{0}\right)$ denote the closed, convex set in the space of $m \times m$ matrices which is the limit, as $c \rightarrow \pm \infty$, of the nested collection of sets $D\left(z, c, x_{0}\right)$ given in Definition 2.5. $D_{ \pm}\left(z, x_{0}\right)$ is said to be a limiting disk. Elements of $D_{ \pm}\left(z, x_{0}\right)$ are denoted by $M_{ \pm}\left(z, x_{0}\right) \in \mathbb{C}^{m \times m}$.

In light of the containment described in 2.29 , for $c \neq x_{0}$ and $z \in \mathbb{C} \backslash \mathbb{R}$,

$$
D_{ \pm}\left(z, x_{0}\right) \subseteq D\left(z, c, x_{0}\right) .
$$

Given its characterization in the second paragraph following Definition 2.5, we see that $D_{ \pm}\left(z, x_{0}\right)$ consists of those matrices $M\left(z, c, x_{0}, \beta\right)$ where $\beta$ satisfies (2.16). In particular,

$$
M_{ \pm}\left(z, x_{0}\right)=M\left(z, c, x_{0}, \beta\right)
$$

for an appropriate choice of $\beta$.

When $D_{ \pm}\left(z, x_{0}\right)$ is a singleton matrix, the system (2.4) is said to be in the limit point (l.p.) case at $\pm \infty$. When $D_{ \pm}\left(z, x_{0}\right)$ has nonempty interior, then (2.4) is said to be in the limit circle (l.c.) case at $\pm \infty$. Indeed, for the case $m=1$, the limit circle case corresponds to $D_{ \pm}\left(z, x_{0}\right)$ being a disk in $\mathbb{C}$.

These apparent geometric properties for the disk correspond to analytic properties for the solutions of the Hamiltonian system (2.4), (2.5). To recall this correspondence, we introduce the following spaces in which we assume that $-\infty \leq a<b \leq \infty$,

$$
\begin{aligned}
L_{A}^{2}((a, b)) & =\left\{\phi:(a, b) \rightarrow \mathbb{C}^{2 m} \mid \int_{a}^{b} d x(\phi(x), A \phi(x))_{\mathbb{C}^{2 m}}<\infty\right\}, \\
N(z, \infty) & =\left\{\phi \in L_{A}^{2}((c, \infty)) \mid J \phi^{\prime}=(z A+B) \phi \text { a.e. on }(c, \infty)\right\}, \\
N(z,-\infty) & =\left\{\phi \in L_{A}^{2}((-\infty, c)) \mid J \phi^{\prime}=(z A+B) \phi \text { a.e. on }(-\infty, c)\right\},
\end{aligned}
$$

for some $c \in \mathbb{R}$ and $z \in \mathbb{C}$. (Here $(\phi, \psi)_{\mathbb{C}^{n}}=\sum_{j=1}^{n} \bar{\phi}_{j} \psi_{j}$ denotes the standard scalar product in $\mathbb{C}^{n}$, abbreviating $\chi \in \mathbb{C}^{n}$ by $\chi=\left(\chi_{1}, \ldots, \chi_{n}\right)^{t}$.) Both dimensions of the spaces in (2.32b) and 2.32c), $\operatorname{dim}_{\mathbb{C}}(N(z, \infty))$ and $\operatorname{dim}_{\mathbb{C}}(N(z,-\infty))$, are constant for $z \in \mathbb{C}_{ \pm}=\{\zeta \in \mathbb{C} \mid \pm \operatorname{Im}(\zeta)>0\}$ (see, e.g., $[12]$, 78 ). One then observes that the Hamiltonian system (2.4) is in the limit point case at $\pm \infty$ whenever

$$
\operatorname{dim}_{\mathbb{C}}(N(z, \pm \infty))=m \text { for all } z \in \mathbb{C} \backslash \mathbb{R}
$$

and in the limit circle case at $\pm \infty$ whenever

$$
\operatorname{dim}_{\mathbb{C}}(N(z, \pm \infty))=2 m \text { for all } z \in \mathbb{C} .
$$

Next we recall the fact that the Dirac-type systems considered in this paper are always in the limit point case at $\pm \infty$.

Lemma 2.9. Assume Hypothesis 2.1. Then the limit point case holds for Diractype systems (i.e., for $A(x)=I_{2 m}$ in (2.4)) at $\pm \infty$. 
Lemma 2.9, under varying sets of assumptions on $B(x)$, is well-known to experts in the field. For instance, in the case $m=1$ and with $B_{1,2}(x)=B_{2,1}(x)$ this fact can be found in 139 . For $B \in C(\mathbb{R})^{2 m \times 2 m}$ and a more general constant matrix $A$, this result is proven in [92] (their proof, however, extends to the current $B \in$ $L_{\text {loc }}^{1}(\mathbb{R})$ case). More generally, multi-dimensional Dirac operators with $L_{\text {loc }}^{2}\left(\mathbb{R}^{n}\right)$ type coefficients (and additional conditions) can be found in 95. A short proof of Lemma 2.9 can be found in [37]. After completion of this paper we became aware of a recent preprint by Lesch and Malamud [93] which provides a thorough study of self-adjointness questions for more general Hamiltonian systems than those considered in this paper.

In either the limit point or limit circle cases, $M_{ \pm}\left(z, x_{0}\right) \in \partial D_{ \pm}\left(z, x_{0}\right)$ is said to be a half-line Weyl-Titchmarsh matrix. (In the l.p. case one of course has $D_{ \pm}\left(z, x_{0}\right)=\partial D_{ \pm}\left(z, x_{0}\right)$.) $\quad$ Each such matrix is associated with the construction of a self-adjoint operator acting on $L_{A}^{2}\left(\left[x_{0}, \pm \infty\right)\right) \cap \mathrm{AC}\left(\left[x_{0}, \pm \infty\right)\right)$ for the Hamiltonian system (2.4), (2.5). However, for those intermediate cases where $m<\operatorname{dim}_{\mathbb{C}}(N(z, \pm \infty))<2 m$, Hinton and Schneider have shown that not every element of the boundary $\partial D_{ \pm}\left(z, x_{0}\right)$ of $D_{ \pm}\left(z, x_{0}\right)$ is a half-line Weyl-Titchmarsh matrix, and have characterized those elements of the boundary that are (cf. [66], 67).

For later reference we summarize the principal results on $M_{ \pm}\left(z, x_{0}\right)$ in the following theorem.

Theorem 2.10 (61], 68], 69], [72], 82). Assume Hypothesis 2.1 and suppose that $z \in \mathbb{C} \backslash \mathbb{R}, x_{0} \in \mathbb{R}$. Then

(i) $\pm M_{ \pm}\left(z, x_{0}\right)$ is an $m \times m$ matrix-valued Herglotz function of maximal rank. In particular,

$$
\begin{gathered}
\operatorname{Im}\left( \pm M_{ \pm}\left(z, x_{0}\right)\right)>0, \quad z \in \mathbb{C}_{+}, \\
M_{ \pm}\left(\bar{z}, x_{0}\right)=M_{ \pm}\left(z, x_{0}\right)^{*}, \\
\operatorname{rank}\left(M_{ \pm}\left(z, x_{0}\right)\right)=m, \\
\lim _{\varepsilon \downarrow 0} M_{ \pm}\left(\lambda+i \varepsilon, x_{0}\right) \text { exists for a.e. } \lambda \in \mathbb{R} .
\end{gathered}
$$

Local singularities of $\pm M_{ \pm}\left(z, x_{0}\right)$ and $\mp M_{ \pm}\left(z, x_{0}\right)^{-1}$ are necessarily real and at most of first order in the sense that

$$
\begin{aligned}
& \mp \lim _{\epsilon \downarrow 0}\left(i \epsilon M_{ \pm}\left(\lambda+i \epsilon, x_{0}\right)\right) \geq 0, \quad \lambda \in \mathbb{R}, \\
& \pm \lim _{\epsilon \downarrow 0}\left(\frac{i \epsilon}{M_{ \pm}\left(\lambda+i \epsilon, x_{0}\right)}\right) \geq 0, \quad \lambda \in \mathbb{R} .
\end{aligned}
$$

(ii) $\pm M_{ \pm}\left(z, x_{0}\right)$ admit the representation

$$
\pm M_{ \pm}\left(z, x_{0}\right)=F_{ \pm}\left(x_{0}\right)+\int_{\mathbb{R}} d \Omega_{ \pm}\left(\lambda, x_{0}\right)\left((\lambda-z)^{-1}-\lambda\left(1+\lambda^{2}\right)^{-1}\right),
$$

where

$$
F_{ \pm}\left(x_{0}\right)=F_{ \pm}\left(x_{0}\right)^{*}, \quad \int_{\mathbb{R}}\left\|d \Omega_{ \pm}\left(\lambda, x_{0}\right)\right\|_{\mathbb{C}^{m \times m}}\left(1+\lambda^{2}\right)^{-1}<\infty
$$

and

$$
\Omega_{ \pm}\left((\lambda, \mu], x_{0}\right)=\lim _{\delta \downarrow 0} \lim _{\varepsilon \downarrow 0} \frac{1}{\pi} \int_{\lambda+\delta}^{\mu+\delta} d \nu \operatorname{Im}\left( \pm M_{ \pm}\left(\nu+i \varepsilon, x_{0}\right)\right)
$$


(iii) Define the $2 m \times m$ matrices

$$
\begin{aligned}
\Psi_{ \pm}\left(z, x, x_{0}\right) & =\left(\begin{array}{l}
\psi_{ \pm, 1}\left(z, x, x_{0}\right) \\
\psi_{ \pm, 2}\left(z, x, x_{0}\right)
\end{array}\right) \\
& =\left(\begin{array}{ll}
\theta_{1}\left(z, x, x_{0}\right) & \phi_{1}\left(z, x, x_{0}\right) \\
\theta_{2}\left(z, x, x_{0}\right) & \phi_{2}\left(z, x, x_{0}\right)
\end{array}\right)\left(\begin{array}{c}
I_{m} \\
M_{ \pm}\left(z, x_{0}\right)
\end{array}\right),
\end{aligned}
$$

then

$$
\operatorname{Im}\left(M_{ \pm}\left(z, x_{0}\right)\right)=\operatorname{Im}(z) \int_{x_{0}}^{ \pm \infty} d x \Psi_{ \pm}\left(z, x, x_{0}\right)^{*} A \Psi_{ \pm}\left(z, x, x_{0}\right),
$$

where A has been introduced in (2.2), (2.3).

In order to describe the Green's matrix associated with (2.12), we assume the hypotheses of Theorem 2.10 and introduce

$$
\begin{array}{r}
K\left(z, x, x^{\prime}\right)=\Psi_{\mp}\left(z, x, x_{0}\right)\left[M_{-}\left(z, x_{0}\right)-M_{+}\left(z, x_{0}\right)\right]^{-1} \Psi_{ \pm}\left(\bar{z}, x^{\prime}, x_{0}\right)^{*}, \\
x \lessgtr x^{\prime}, z \in \mathbb{C} \backslash \mathbb{R},
\end{array}
$$

and

$$
\begin{aligned}
M\left(z, x_{0}\right) & =\left(M_{j, j^{\prime}}\left(z, x_{0}\right)\right)_{j, j^{\prime}=1,2} \\
& =\left[K\left(z, x_{0}, x_{0}+0\right)+K\left(z, x_{0}, x_{0}-0\right)\right] / 2, \quad z \in \mathbb{C} \backslash \mathbb{R}, \\
M_{1,1}\left(z, x_{0}\right) & =\left[M_{-}\left(z, x_{0}\right)-M_{+}\left(z, x_{0}\right)\right]^{-1}, \\
M_{1,2}\left(z, x_{0}\right) & =2^{-1}\left[M_{-}\left(z, x_{0}\right)-M_{+}\left(z, x_{0}\right)\right]^{-1}\left[M_{-}\left(z, x_{0}\right)+M_{+}\left(z, x_{0}\right)\right], \\
M_{2,1}\left(z, x_{0}\right) & =2^{-1}\left[M_{-}\left(z, x_{0}\right)+M_{+}\left(z, x_{0}\right)\right]\left[M_{-}\left(z, x_{0}\right)-M_{+}\left(z, x_{0}\right)\right]^{-1}, \\
M_{2,2}\left(z, x_{0}\right) & =M_{ \pm}\left(z, x_{0}\right)\left[M_{-}\left(z, x_{0}\right)-M_{+}\left(z, x_{0}\right)\right]^{-1} M_{\mp}\left(z, x_{0}\right) .
\end{aligned}
$$

Next let $\phi \in L_{A}^{2}(\mathbb{R})$ and consider

$$
J \psi^{\prime}(z, x)=(z A+B(x)) \psi(z, x)+A \phi(x), \quad z \in \mathbb{C} \backslash \mathbb{R}
$$

for a.e. $x \in \mathbb{R}$. Then $(2.48)$ has a unique solution $\psi(z, \cdot) \in L_{A}^{2}(\mathbb{R}) \cap \mathrm{AC}_{\text {loc }}(\mathbb{R})^{2 m}$ given by [68], 70]

$$
\psi(z, x)=\int_{\mathbb{R}} d x^{\prime} K\left(z, x, x^{\prime}\right) A \phi\left(x^{\prime}\right) .
$$

In the following we associate an operator $T$ in $L^{2}(\mathbb{R})^{m}$, respectively, $L^{2}(\mathbb{R})^{2 m}$ with the Hamiltonian system (2.4) denoted by

$$
T= \begin{cases}H & \text { if } A=\left(\begin{array}{cc}
I_{m} & 0 \\
0 & 0
\end{array}\right), \\
D & \text { if } A=I_{2 m} .\end{cases}
$$

Here $H$ is defined by

$$
\left((H-z)^{-1} f\right)(x)=\int_{\mathbb{R}} d x^{\prime} K_{1,1}\left(z, x, x^{\prime}\right) f\left(x^{\prime}\right), \quad z \in \mathbb{C} \backslash \mathbb{R}, f \in L^{2}(\mathbb{R})^{m},
$$

and this results in the following matrix-valued Schrödinger operator in $L^{2}(\mathbb{R})^{m}$,

$$
\begin{aligned}
H & =-I_{m} \frac{d^{2}}{d x^{2}}+Q \\
\operatorname{dom}(H) & =\left\{g \in L^{2}(\mathbb{R})^{m} \mid g, g^{\prime} \in \mathrm{AC}_{\mathrm{loc}}(\mathbb{R})^{m} ; \text { s. s.-a. b. c. at } \pm \infty ;\right.
\end{aligned}
$$




$$
\left.\left(-I_{m} g^{\prime \prime}+Q g\right) \in L^{2}(\mathbb{R})^{m}\right\}
$$

where "s. s.-a. b. c. at $\pm \infty$ " denotes a separated self-adjoint boundary condition at $+\infty$ and/or $-\infty$ (if any) induced by $M_{+}\left(z, x_{0}\right)$ and/or $M_{-}\left(z, x_{0}\right)$. Here $K_{1,1}\left(z, x, x^{\prime}\right)$ is the left upper $m \times m$ submatrix of $K\left(z, x, x^{\prime}\right)$, obtained from the partitioning $K\left(z, x, x^{\prime}\right)=\left(K_{j, k}\left(z, x, x^{\prime}\right)\right)_{j, k=1}^{2}$. Similarly, $D$ defined by

$$
\left((D-z)^{-1} \psi\right)(x)=\int_{\mathbb{R}} d x^{\prime} K\left(z, x, x^{\prime}\right) \psi\left(x^{\prime}\right), \quad z \in \mathbb{C} \backslash \mathbb{R}, \psi \in L^{2}(\mathbb{R})^{2 m},
$$

represents the following Dirac-type operator in $L^{2}(\mathbb{R})^{2 m}$,

$$
\begin{aligned}
D & =J \frac{d}{d x}-B, \\
\operatorname{dom}(D) & =\left\{\phi \in L^{2}(\mathbb{R})^{2 m} \mid \phi \in \mathrm{AC}_{\mathrm{loc}}(\mathbb{R})^{2 m} ;\left(J \phi^{\prime}-B \phi\right) \in L^{2}(\mathbb{R})^{2 m}\right\},
\end{aligned}
$$

where we took into account the limit point property of Dirac-type operators as described in Lemma 2.9. Thus, $H$ and $D$ are self-adjoint operators in $L^{2}(\mathbb{R})^{m}$ and $L^{2}(\mathbb{R})^{2 m}$, respectively.

The basic results on $M\left(z, x_{0}\right)$ then read as follows.

Theorem 2.11 (61], 68], 69], [72], 82). Assume Hypothesis 2.1 and suppose that $z \in \mathbb{C} \backslash \mathbb{R}, x_{0} \in \mathbb{R}$. Then

(i) $M\left(z, x_{0}\right)$ is a matrix-valued Herglotz function of rank $2 m$ with representation

$$
M\left(z, x_{0}\right)=F\left(x_{0}\right)+\int_{\mathbb{R}} d \Omega\left(\lambda, x_{0}\right)\left((\lambda-z)^{-1}-\lambda\left(1+\lambda^{2}\right)^{-1}\right),
$$

where

$$
F\left(x_{0}\right)=F\left(x_{0}\right)^{*}, \quad \int_{\mathbb{R}}\left\|d \Omega\left(\lambda, x_{0}\right)\right\|_{\mathbb{C}^{2 m \times 2 m}}\left(1+\lambda^{2}\right)^{-1}<\infty
$$

and

$$
\Omega\left((\lambda, \mu], x_{0}\right)=\lim _{\delta \downarrow 0} \lim _{\varepsilon \downarrow 0} \frac{1}{\pi} \int_{\lambda+\delta}^{\mu+\delta} d \nu \operatorname{Im}\left(M\left(\nu+i \varepsilon, x_{0}\right)\right) .
$$

(ii) $z \in \rho(T)$ if and only if $M\left(z, x_{0}\right)$ is holomorphic near $z$.

The fundamental uniqueness theorem for $T$ in terms of $M\left(z, x_{0}\right)$ then reads as follows.

Theorem 2.12 (36], [37, 60], [92], 116]). Let $x_{0} \in \mathbb{R}$ and assume Hypothesis 2.9. Then $M\left(z, x_{0}\right)$ for all $z \in \mathbb{C}_{+}$uniquely determines $T$. In particular, in the case of the Schrödinger operator $H, M\left(z, x_{0}\right), z \in \mathbb{C}_{+}$, uniquely determines $Q(x)$ for a.e. $x \in \mathbb{R}$, and in the case of the Dirac-type operator $D, M\left(z, x_{0}\right), z \in \mathbb{C}_{+}$, uniquely determines $B(x)=\left(\begin{array}{cc}B_{1,1}(x) & B_{1,2}(x) \\ B_{1,2}(x) & -B_{1,1}(x)\end{array}\right)$ for a.e. $x \in \mathbb{R}$.

In the context of Schrödinger operators, Theorem 2.12 represents the matrixvalued extension of the celebrated Borg-Marchenko uniqueness theorem 24, [104, 105 (see also [59], [134) for Schrödinger operators on a half-line in the scalar case $m=1$. The matrix Schrödinger case has been treated by Rofe-Beketov [116] (assuming $Q$ to be continuous on $\mathbb{R}$ ) and more recently in 60 (in the general case $Q \in L^{1}([0, R])^{m \times m}$ for all $\left.R>0\right)$. In the Dirac case, and for $B$ continuous, Theorem 2.12 is contained in [92]. The Dirac result under the weaker Hypothesis 2.2 is due to [37. In the scalar case $m=1$, boundedness of $B$ is immaterial for the uniqueness result to hold in Theorem 2.12 (cf. the discussion in [37]). However, 
for $m \geq 2$, the corresponding uniqueness result appears to have been proved only assuming $B \in L_{\text {loc }}^{\infty}(\mathbb{R})^{2 m \times 2 m}$ thus far. This is the reason why we added the local essentially boundedness assumption of $B$ to Hypothesis 2.2. As soon as one succeeds in removing this $L_{\mathrm{loc}}^{\infty}$-assumption of $B$ in Theorem 2.12, it can be removed everywhere in this paper.

The recent new results on local Borg-Marchenko uniqueness theorems for matrixvalued Schrödinger and Dirac-type operators on a half-line in [37], [60] then yield the following local extension of Theorem 2.12.

Theorem 2.13 (37, [60]). Suppose $B_{j}, j=1,2$, satisfy Hypothesis 2.9 and let $a>0, a_{ \pm}>0$, and $x_{0} \in \mathbb{R}$.

(i) Denote by $M_{j, \pm}\left(z, x_{0}\right), j=1,2$ the $m \times m$ Weyl-Titchmarsh matrices associated with the corresponding Hamiltonian systems (2.4) on $\left(x_{0}, \pm \infty\right)$. In the Schrödinger case assume that

$$
\left\|M_{1, \pm}\left(z, x_{0}\right)-M_{2, \pm}\left(z, x_{0}\right)\right\|_{\mathbb{C}^{m \times m}} \underset{|z| \rightarrow \infty}{=} O\left(e^{-2 \operatorname{Im}\left(z^{1 / 2}\right) a_{ \pm}}\right)
$$

along a ray with $0<\arg (z)<\pi$. In the Dirac-type case suppose that

$$
\left\|M_{1, \pm}\left(z, x_{0}\right)-M_{2, \pm}\left(z, x_{0}\right)\right\|_{\mathbb{C}^{m \times m}} \underset{|z| \rightarrow \infty}{=} O\left(e^{-2 \operatorname{Im}(z) a_{ \pm}}\right)
$$

along a ray with $0<\arg (z)<\pi / 2$ and along a ray with $\pi / 2<\arg (z)<\pi$. Then, in either case,

$$
B_{1}(x)=B_{2}(x) \text { for a.e. } x \in\left[x_{0}, x_{0} \pm a_{ \pm}\right] .
$$

(ii) Denote by $M_{j}\left(z, x_{0}\right), j=1,2$ the $2 m \times 2 m$ Weyl-Titchmarsh matrices associated with the corresponding Hamiltonian systems (2.4) on $\mathbb{R}$. In the Schrödinger case assume that

$$
\left\|M_{1}\left(z, x_{0}\right)-M_{2}\left(z, x_{0}\right)\right\|_{\mathbb{C}^{2 m \times 2 m}} \underset{|z| \rightarrow \infty}{=} O\left(e^{-2 \operatorname{Im}\left(z^{1 / 2}\right) a}\right)
$$

along a ray with $0<\arg (z)<\pi$. In the Dirac-type case suppose that

$$
\left\|M_{1}\left(z, x_{0}\right)-M_{2}\left(z, x_{0}\right)\right\|_{\mathbb{C}^{2 m \times 2 m}} \underset{|z| \rightarrow \infty}{=} O\left(e^{-2 \operatorname{Im}\left(z^{1 / 2}\right) a}\right)
$$

along a ray with $0<\arg (z)<\pi / 2$ and along a ray with $\pi / 2<\arg (z)<\pi$. Then, in either case,

$$
B_{1}(x)=B_{2}(x) \text { for a.e. } x \in\left[x_{0}-a, x_{0}+a\right] .
$$

Proof. In the context of Schrödinger operators, case (i) is proved in 60; in the corresponding Dirac-type situation case (i) is proved in 37. Case (ii) then follows by combining (2.47) and case (i), taking into account the asymptotic expansions (along any ray with $0<\arg (z)<\pi$ )

$$
M_{ \pm}\left(z, x_{0}\right) \underset{|z| \rightarrow \infty}{=} \pm i z^{1 / 2} I_{m}+o(1)
$$

in the case of Schrödinger operators (cf. [36]) and

$$
M_{ \pm}\left(z, x_{0}\right) \underset{|z| \rightarrow \infty}{=} \pm i I_{m}+o(1)
$$

in the case of Dirac-type operators (cf. [37]). 
In the case of scalar Schrödinger operators, Theorem 2.13(i) is due to Simon 134. An alternative proof was presented in [60], and more recently in [16]. Most recently, Alexander Sakhnovich kindly informed us that his integral representation of the Weyl-Titchmarsh matrix in 120 can be used to derive asymptotic expansions for the Weyl-Titchmarsh matrix and its associated matrix-valued spectral function, and also yields a result analogous to Theorem 2.13 (i) for a certain class of canonical systems. Moreover, in the case of skew-adjoint Dirac-type systems, similar results are discussed in [121 and applied to the nonlinear Schrödinger equation on a halfaxis.

\section{Uniqueness Results for Schrödinger and Dirac-Type Operators}

In this section we prove our uniqueness results for matrix-valued Schrödinger and Dirac-type operators.

Since in the case of Schrödinger operators, $B(x)$ is of a very special nature,

$$
B(x)=\left(\begin{array}{cc}
-Q(x) & 0 \\
0 & I_{m}
\end{array}\right), \quad Q(x)^{*}=Q(x),
$$

and only the $m \times m$ submatrix $B_{1,1}=-Q(x)$ contains information on $Q(x)$, we will focus on the $m \times m$ submatrix $M_{1,1}\left(z, x_{0}\right)$ of $M\left(z, x_{0}\right)$ in (2.47) and (2.55), (2.56). By (2.46) and (2.51) one infers that the Green's matrix $G\left(z, x, x^{\prime}\right)$ of $H$ is given by

$$
\begin{aligned}
G\left(z, x, x^{\prime}\right) & =K_{1,1}\left(z, x, x^{\prime}\right) \\
& =\psi_{\mp, 1}\left(z, x, x_{0}\right)\left[M_{-}\left(z, x_{0}\right)-M_{+}\left(z, x_{0}\right)\right]^{-1} \psi_{ \pm, 1}\left(\bar{z}, x^{\prime}, x_{0}\right)^{*}, \\
& x \lesseqgtr x^{\prime}, z \in \mathbb{C} \backslash \mathbb{R},
\end{aligned}
$$

where the $m \times m$ matrices $\psi_{ \pm, j}\left(z, x, x_{0}\right)$ are defined in 2.44 , that is,

$$
\psi_{ \pm, j}\left(z, x, x_{0}\right)=\theta_{j}\left(z, x, x_{0}\right)+\phi_{j}\left(z, x, x_{0}\right) M_{ \pm}\left(z, x_{0}\right), \quad j=1,2 .
$$

For future purpose we abbreviate the diagonal Green's function $G(z, x, x)$ of $H$ by $g(z, x)$, that is,

$$
g(z, x)=G(z, x, x), \quad z \in \mathbb{C} \backslash \mathbb{R}, x \in \mathbb{R} .
$$

Moreover, since "diagonal elements" of matrix-valued Herglotz functions are (lowerdimensional) matrix-valued Herglotz functions (cf., e.g., 61), $M_{1,1}\left(z, x_{0}\right)=g\left(z, x_{0}\right)$ is an $m \times m$ matrix-valued Herglotz function satisfying

$$
\begin{aligned}
M_{1,1}\left(z, x_{0}\right) & =g\left(z, x_{0}\right)=K_{1,1}\left(z, x_{0}, x_{0}\right) \\
& =\left[M_{-}\left(z, x_{0}\right)-M_{+}\left(z, x_{0}\right)\right]^{-1} \\
& =F_{1,1}\left(x_{0}\right)+\int_{\mathbb{R}} d \Omega_{1,1}\left(\lambda, x_{0}\right)\left((\lambda-z)^{-1}-\lambda\left(1+\lambda^{2}\right)^{-1}\right),
\end{aligned}
$$

where

$$
F_{1,1}\left(x_{0}\right)=F_{1,1}\left(x_{0}\right)^{*}, \quad \int_{\mathbb{R}}\left\|d \Omega_{1,1}\left(\lambda, x_{0}\right)\right\|_{\mathbb{C}^{m \times m}}\left(1+\lambda^{2}\right)^{-1}<\infty
$$

and

$$
\Omega_{1,1}\left((\lambda, \mu], x_{0}\right)=\lim _{\delta \downarrow 0} \lim _{\varepsilon \downarrow 0} \frac{1}{\varepsilon} \int_{\lambda+\delta}^{\mu+\delta} d \nu \operatorname{Im}\left(M_{1,1}\left(\nu+i \varepsilon, x_{0}\right)\right) .
$$

In complete analogy to the Schrödinger case, we also introduce

$$
g\left(z, x_{0}\right)=M_{1,1}\left(z, x_{0}\right)=\left[M_{-}\left(z, x_{0}\right)-M_{+}\left(z, x_{0}\right)\right]^{-1}, \quad z \in \mathbb{C} \backslash \mathbb{R}
$$


in the case of Dirac-type operators $D$ and recall that $B(x)$ is now of the type

$$
\begin{aligned}
& B(x)=\left(\begin{array}{ll}
B_{1,1}(x) & B_{1,2}(x) \\
B_{2,1}(x) & B_{2,2}(x)
\end{array}\right), \\
& \text { with } B_{1,2}(x)^{*}=B_{2,1}(x), B_{j, j}(x)^{*}=B_{j, j}(x), j=1,2 .
\end{aligned}
$$

Next, we provide a quick derivation of the matrix Riccati-type equations satisfied by $M_{ \pm}(z, x)$ in the Schrödinger and Dirac cases. This result is not new (see, e.g., [38, [55], 75]). We only mention its proof in some detail to reach a certain level of completeness and since it is a fundamental ingredient in our principal uniqueness result, Theorem 3.7.

Lemma 3.1. Assume Hypothesis 2.1, $z \in \mathbb{C} \backslash \mathbb{R}$, and $x \in \mathbb{R}$. Then in the case of matrix-valued Schrödinger operators $H, M_{ \pm}(z, x)$ satisfies the standard Riccati-type equation,

$$
M_{ \pm}^{\prime}(z, x)+M_{ \pm}(z, x)^{2}=Q(x)-z I_{m}
$$

for a.e. $x \in \mathbb{R}$. In the case of Dirac-type operators $D, M_{ \pm}(z, x)$ satisfies

$$
\begin{aligned}
& M_{ \pm}^{\prime}(z, x)+z M_{ \pm}(z, x)^{2}+M_{ \pm}(z, x) B_{2,2}(x) M_{ \pm}(z, x)+B_{1,2}(x) M_{ \pm}(z, x) \\
& +M_{ \pm}(z, x) B_{2,1}(x)=-B_{1,1}(x)-z I_{m}
\end{aligned}
$$

for a.e. $x \in \mathbb{R}$.

Proof. In order to prove 3.12 ), let

$$
\hat{\Psi}\left(z, x, x_{0}\right)=\left(\begin{array}{ll}
\psi_{-, 1}\left(z, x, x_{0}\right) & \psi_{+, 1}\left(z, x, x_{0}\right) \\
\psi_{-, 2}\left(z, x, x_{0}\right) & \psi_{+, 2}\left(z, x, x_{0}\right)
\end{array}\right), \quad z \in \mathbb{C} \backslash \mathbb{R}
$$

be the fundamental system of solutions of (2.12) as defined in (2.44) and observe that

$$
\psi_{+, 2}\left(z, x, x_{0}\right)=\psi_{+, 1}^{\prime}\left(z, x, x_{0}\right)
$$

in the Schrödinger operator case. Thus, any nonnormalized solutions $\tilde{\psi}_{ \pm, 1}(z, \cdot) \in$ $L^{2}(( \pm \infty, c))^{m \times m}, c \in \mathbb{R}$ of $-\psi_{1}^{\prime \prime}+Q \psi_{1}=z \psi_{1}$ for $z \in \mathbb{C} \backslash \mathbb{R}$ are of the type

$$
\tilde{\psi}_{ \pm, 1}(z, x)=\psi_{ \pm, 1}\left(z, x, x_{0}\right) C_{ \pm}
$$

for some nonsingular $m \times m$ matrices $C_{ \pm}$. Thus,

$$
\tilde{\psi}_{ \pm, 1}\left(z, x_{0}\right)=C_{ \pm}, \quad \tilde{\psi}_{ \pm, 1}^{\prime}\left(z, x_{0}\right)=M_{ \pm}\left(z, x_{0}\right) C_{ \pm}
$$

by 2.15$)$ and (2.44). In particular,

$$
M_{ \pm}\left(z, x_{0}\right)=\tilde{\psi}_{ \pm, 1}^{\prime}\left(z, x_{0}\right) \tilde{\psi}_{ \pm, 1}\left(z, x_{0}\right)^{-1}, \quad z \in \mathbb{C} \backslash \mathbb{R}
$$

is independent of the normalization chosen for $\tilde{\psi}_{ \pm, 1}\left(z, x_{0}\right)$. Varying the reference point $x_{0} \in \mathbb{R}$ then yields (3.12). Similarly, in order to derive (3.13), let

$$
\hat{\Psi}\left(z, x, x_{0}\right)=\left(\begin{array}{ll}
\psi_{-, 1}\left(z, x, x_{0}\right) & \psi_{+, 1}\left(z, x, x_{0}\right) \\
\psi_{-, 2}\left(z, x, x_{0}\right) & \psi_{+, 2}\left(z, x, x_{0}\right)
\end{array}\right), \quad z \in \mathbb{C}_{+}
$$

be the fundamental system of solutions of (2.12) as defined in (2.44). Any other fundamental system of (2.12) of the form

$$
\tilde{\Psi}(z, x)=\left(\begin{array}{cc}
\tilde{\psi}_{-, 1}(z, x) & \tilde{\psi}_{+, 1}(z, x) \\
\tilde{\psi}_{-, 2}(z, x) & \tilde{\psi}_{+, 2}(z, x)
\end{array}\right), \quad z \in \mathbb{C}_{+},
$$


with $\tilde{\Psi}_{ \pm}(z, x)=\left(\tilde{\psi}_{ \pm, 1}(z, x), \tilde{\psi}_{ \pm, 2}(z, x)\right)^{t}$ a basis of $N(z, \pm \infty)$ for $z \in \mathbb{C}_{+}$, necessarily must be of the form

$$
\tilde{\Psi}(z, x)=\hat{\Psi}\left(z, x, x_{0}\right) C .
$$

Here $C$ is of the type

$$
C=\left(\begin{array}{cc}
C_{+} & 0 \\
0 & C_{-}
\end{array}\right)
$$

and $C_{ \pm}$are invertible $m \times m$ matrices. In particular,

$$
\tilde{\psi}_{-, j}(z, x)=\psi_{-, j}\left(z, x, x_{0}\right) C_{-}, \quad \tilde{\psi}_{+, j}(z, x)=\psi_{+, j}\left(z, x, x_{0}\right) C_{+}, j=1,2
$$

and thus,

$$
\tilde{\psi}_{ \pm, 1}\left(z, x_{0}\right)=C_{ \pm}, \quad \tilde{\psi}_{ \pm, 2}\left(z, x_{0}\right)=M_{ \pm}\left(z, x_{0}\right) C_{ \pm}
$$

by (2.15) and (2.44). Consequently,

$$
M_{ \pm}\left(z, x_{0}\right)=\tilde{\psi}_{ \pm, 2}\left(z, x_{0}\right) \tilde{\psi}_{ \pm, 1}\left(z, x_{0}\right)^{-1}, \quad z \in \mathbb{C}_{+}
$$

is independent of the normalization chosen for $\tilde{\psi}_{ \pm, j}$. Varying $x_{0} \in \mathbb{R}$ one verifies (3.13).

Corollary 3.2. Assume Hypothesis 2.1, $z \in \mathbb{C} \backslash \mathbb{R}$, and $x \in \mathbb{R}$. Moreover, define

$$
g(z, x)=\left[M_{-}(z, x)-M_{+}(z, x)\right]^{-1}, \quad z \in \mathbb{C} \backslash \mathbb{R}, x \in \mathbb{R}
$$

as in (3.4) and (3.10). Then in the case of matrix-valued Schrödinger operators, the diagonal Green's matrix $g(z, x)$ of $H$, satisfies

$$
\begin{aligned}
g^{\prime}(z, x) & =g(z, x) M_{+}(z, x)+M_{-}(z, x) g(z, x), \\
g^{\prime}(z, x) \mp I_{m} & =g(z, x) M_{ \pm}(z, x)+M_{ \pm}(z, x) g(z, x)
\end{aligned}
$$

for all $x \in \mathbb{R}$. In the case of Dirac-type operators $D, g(z, x)$ satisfies

$$
\begin{aligned}
g^{\prime}(z, x)= & z g(z, x) M_{+}(z, x)+z M_{-}(z, x) g(z, x)-g(z, x) M_{ \pm}(z, x) B_{1,1}(x) \\
& -B_{1,1}(x) M_{\mp}(z, x) g(z, x)+g(z, x) B_{1,2}(x)+B_{1,2}(x) g(z, x)
\end{aligned}
$$

for a.e. $x \in \mathbb{R}$.

Proof. In order to prove (3.27) one only needs to observe

$$
\begin{aligned}
(d / d x)\left(g(z, x)^{-1}\right)=-g(z, x)^{-1} g^{\prime}(z, x) g(z, x)^{-1} \\
=M_{-}^{\prime}(z, x)-M_{+}^{\prime}(z, x) \\
=M_{+}(z, x)^{2}-M_{-}(z, x)^{2} \\
=M_{+}(z, x)\left[M_{+}(z, x)-M_{-}(z, x)\right] \\
\quad+\left[M_{+}(z, x)-M_{-}(z, x)\right] M_{-}(z, x) \\
=-M_{+}(z, x) g(z, x)^{-1}-g(z, x)^{-1} M_{-}(z, x)
\end{aligned}
$$

for a.e. $x \in \mathbb{R}$, using $(3.12)$ and $(3.26)$. By continuity of the right-hand side of (3.30) with respect to $x,(3.30)$ extends to all $x \in \mathbb{R}$. Equation (3.30) then proves (3.27), and using (3.26) again, (3.27) proves (3.28). Similarly, to prove (3.29), one computes

$$
\begin{aligned}
g^{\prime}(z, x)= & -\left[M_{-}(z, x)-M_{+}(z, x)\right]^{-1}\left[M_{-}^{\prime}(z, x)-M_{+}^{\prime}(z, x)\right] \times \\
& \times\left[M_{-}(z, x)-M_{+}(z, x)\right]^{-1}
\end{aligned}
$$




$$
\begin{aligned}
= & -\left[M_{-}(z, x)-M_{+}(z, x)\right]^{-1}\left[z M_{+}(z, x)^{2}-z M_{-}(z, x)^{2}\right. \\
& -M_{+}(z, x) B_{1,1}(x) M_{+}(z, x)+M_{-}(z, x) B_{1,1}(x) M_{-}(z, x) \\
& +B_{1,2}(x) M_{+}(z, x)-B_{1,2}(x) M_{-}(z, x)+M_{+}(z, x) B_{1,2}(x) \\
& \left.-M_{-}(z, x) B_{1,2}(x)\right]\left[M_{-}(z, x)-M_{+}(z, x)\right]^{-1} \\
= & z g(z, x) M_{+}(z, x)+z M_{-}(z, x) g(z, x) \\
& +\left[M_{-}(z, x)-M_{+}(z, x)\right]^{-1}\left[M_{+}(z, x) B_{1,1}(x) M_{+}(z, x)\right. \\
& +M_{ \pm}(z, x) B_{1,1}(x) M_{\mp}(z, x)-M_{ \pm}(z, x) B_{1,1}(x) M_{\mp}(z, x) \\
& \left.+M_{-}(z, x) B_{1,1}(x) M_{-}(z, x)\right]\left[M_{-}(z, x)-M_{+}(z, x)\right]^{-1} \\
& +g(z, x) B_{1,2}(x)+B_{1,2}(x) g(z, x) \\
= & z g(z, x) M_{+}(z, x)+z M_{-}(z, x) g(z, x)-g(z, x) M_{ \pm}(z, x) B_{1,1}(x) \\
& -B_{1,1}(x) M_{\mp}(z, x) g(z, x)+g(z, x) B_{1,2}(x)+B_{1,2}(x) g(z, x)
\end{aligned}
$$

for a.e. $x \in \mathbb{R}$, using (3.13) and (3.26).

Denoting by $\operatorname{spec}(A)$ the spectrum (i.e., the set of eigenvalues) of an $n \times n$ matrix $A \in \mathbb{C}^{n \times n}$, we next recall a well-known lemma concerning the solution of Sylvester's matrix equation. We also recall that $A$ is said to be accretive if $\operatorname{Re}\left((\underline{x}, A \underline{x})_{\mathbb{C}^{n}}\right) \geq 0$ for all $\underline{x} \in \mathbb{C}^{n}$. Finally, we recall that \|\|$\cdot \| \mid$ is said to be a unitarily invariant norm on $\mathbb{C}^{n \times n}$ if $\|U A V\||=|\|A\| \|$ for all $U, V \in \mathbb{C}^{n \times n}$ unitary and all $A \in \mathbb{C}^{n \times n}$.

Lemma 3.3 (c.f. [22], Theorems VII.2.1 and VII.2.4 and [23]). Assume A, B, C $\mathbb{C}^{n \times n}$ for some $n \in \mathbb{N}$.

(i) Suppose that

$$
\operatorname{spec}(A) \cap \operatorname{spec}(B)=\emptyset .
$$

Then the equation

$$
A X-X B=C
$$

has a unique solution $X \in \mathbb{C}^{n \times n}$. Moreover, suppose $\Gamma$ is a closed counterclockwise oriented Jordan contour such that $\operatorname{spec}(A)$ has winding number +1 and $\operatorname{spec}(B)$ has winding number 0 with respect to $\Gamma$. Then an explicit solution for $X$ in (3.33) is provided by

$$
X=(2 \pi i)^{-1} \oint_{\Gamma} d \zeta(A-\zeta)^{-1} C(B-\zeta)^{-1}
$$

(ii) Assume that $A-(\delta / 2) I_{n}$ and $-B-(\delta / 2) I_{n}$ are accretive. Then (3.33) has a unique solution $X \in \mathbb{C}^{n \times n}$ satisfying

$$
\left\|| | X \left|\left\|\leq \delta^{-1}|| C \mid\right\|\right.\right.
$$

for any unitarily invariant norm $\||\cdot|\|$ on $\mathbb{C}^{n \times n}$.

Without going into details, we note that Lemma 3.3(i) remains valid for bounded operators on a Banach space and also extends to certain cases where $A$ and $B$ are unbounded (cf., e.g., [115)). Similarly, Lemma 3.3.(ii) is proven for maximal accretive operators $A-(\delta / 2) I,-B-(\delta / 2) I$ in a Hilbert space in [23].

Next, we recall one more auxiliary result to prove the principal uniqueness results of this section, Theorems 3.7 and 3.8. Although we are only applying the following result in the special case of $m \times m$ matrices, we state the corresponding facts in the infinite-dimensional context since, in contrast to Lemma 3.3, this appears to be 
a less familiar result. In the following let $\mathcal{H}$ be a complex separable Hilbert space with scalar product $(\cdot, \cdot)_{\mathcal{H}}$ (and norm $\|\cdot\|_{\mathcal{H}}$ ), and denote by $\mathcal{B}(\mathcal{H})$ the Banach space of bounded linear operators on $\mathcal{H}$.

Lemma 3.4 (c.f. [56], Lemma 2.3). Let $M(z)$ be an operator-valued Herglotz function with values in $\mathcal{B}(\mathcal{H})$ (i.e., $M$ is analytic in $\mathbb{C}_{+}$with $\operatorname{Im}(M(z)) \geq 0$ ). If $\left(\operatorname{Im}\left(M\left(z_{0}\right)\right)\right)^{-1} \in \mathcal{B}(\mathcal{H})$ for some $z_{0} \in \mathbb{C}_{+}$, then $(\operatorname{Im}(M(z)))^{-1} \in \mathcal{B}(\mathcal{H})$ for all $z \in \mathbb{C}_{+}$and

$$
\operatorname{spec}(M(z)) \subset \mathbb{C}_{+} \text {and hence } \operatorname{spec}(M(z)) \cap \mathbb{R}=\emptyset .
$$

Proof. Suppose there is a $z_{1} \in \mathbb{C}_{+}$such that $\left(\operatorname{Im}\left(M\left(z_{1}\right)\right)\right)^{-1} \notin \mathcal{B}(\mathcal{H})$. Then there is a sequence $\left\{e_{n}\right\}_{n} \subset \mathcal{H},\left\|e_{n}\right\|_{\mathcal{H}}=1, n \in \mathbb{N}$, such that

$$
\lim _{n \rightarrow \infty}\left\|\operatorname{Im}\left(M\left(z_{1}\right)\right) e_{n}\right\|_{\mathcal{H}}=0
$$

Any Herglotz function $M(z)$ with values in $\mathcal{B}(\mathcal{H})$ admits the representation

$$
M(z)=A+B z+\left.R^{1 / 2}\left(I_{\mathcal{K}}+z L\right)(L-z)^{-1} R^{1 / 2}\right|_{\mathcal{H}},
$$

where $A=A^{*} \in \mathcal{B}(\mathcal{H}), 0 \leq B \in \mathcal{B}(\mathcal{H}), \mathcal{K}$ is a Hilbert space, $\mathcal{K} \subseteq \mathcal{H}, L=L^{*}$ is a self-adjoint operator in $\mathcal{K}$, and $0 \leq R \in \mathcal{B}(\mathcal{K})$ with $\left.R\right|_{\mathcal{K} \ominus \mathcal{H}}=0$. By $(3.38)$,

$$
\begin{aligned}
\operatorname{Im}(M(z)) & =\operatorname{Im}(z)\left(B+\left.R^{1 / 2}\left(I_{\mathcal{K}}+L^{2}\right)\left((L-\operatorname{Re}(z))^{2}+(\operatorname{Im}(z))^{2}\right)^{-1} R^{1 / 2}\right|_{\mathcal{H}}\right) \\
& =\operatorname{Im}(z)\left(B+\left.R^{1 / 2} C(z) R^{1 / 2}\right|_{\mathcal{H}}\right),
\end{aligned}
$$

where $C(z)=\left(I_{\mathcal{K}}+L^{2}\right)\left((L-\operatorname{Re}(z))^{2}+(\operatorname{Im}(z))^{2}\right)^{-1}>0$ is invertible in $\mathcal{K}, C(z)^{-1} \in$ $\mathcal{B}(\mathcal{K}), z \in \mathbb{C}_{+}$. Thus, (3.37) together with (3.39) imply

$$
\lim _{n \rightarrow \infty}\left(\left(e_{n}, B e_{n}\right)_{\mathcal{H}}+\left(\left.R^{1 / 2}\right|_{\mathcal{H}} e_{n},\left.C\left(z_{1}\right) R^{1 / 2}\right|_{\mathcal{H}} e_{n}\right)_{\mathcal{H}}\right)=0
$$

and hence $\lim _{n \rightarrow \infty}\left\|B^{1 / 2} e_{n}\right\|_{\mathcal{H}}=\lim _{n \rightarrow \infty}\left\|B e_{n}\right\|_{\mathcal{H}}=\lim _{n \rightarrow \infty}\left\|\left.R^{1 / 2}\right|_{\mathcal{H}} e_{n}\right\|_{\mathcal{H}}=0$. Applying (3.39) again one infers $\lim _{n \rightarrow \infty}\left\|\operatorname{Im}(M(z)) e_{n}\right\|_{\mathcal{H}}=0$ for all $z \in \mathbb{C}_{+}$, contradicting the hypothesis $\left(\operatorname{Im}\left(M\left(z_{0}\right)\right)\right)^{-1} \in \mathcal{B}(\mathcal{H})$.

Since $\operatorname{Im}(M(z)), z \in \mathbb{C}_{+}$is invertible, the numerical range $W_{z}$ of the operator $M(z)$

$$
W_{z}=\left\{(M(z) e, e)_{\mathcal{H}} \mid e \in \mathcal{H},\|e\|_{\mathcal{H}}=1\right\}
$$

is a subset of the half-plane $\left\{\zeta \in \mathbb{C}_{+} \mid \operatorname{Im}(\zeta) \geq\left\|\left(\operatorname{Im}(M(z))^{-1}\right)\right\|_{\mathcal{B}(\mathcal{H})}^{-1}\right\}$. By the spectral inclusion theorem (see, e.g., [63, Theorem1.2-1]), the spectrum of $M(z)$ is contained in the closure of the numerical range $W_{z}$ and hence (3.36) holds.

Moreover, we will also apply the following elementary result.

Lemma 3.5. Let $A, B \in \mathbb{C}^{n \times n}$ for some $n \in \mathbb{N}$ and

$$
\|A\|_{\mathbb{C}^{n \times n}}<1 / 2, \quad\|B\|_{\mathbb{C}^{n \times n}}<1 / 2
$$

for some matrix norm $\|\cdot\|$ on $\mathbb{C}^{n \times n}$. Then the matrix-valued Riccati equation

$$
Y=Y A Y+B
$$

has a unique solution $X \in \mathbb{C}^{n \times n} \cap \bar{K}$, where $\bar{K}=\left\{X \in \mathbb{C}^{n \times n} \mid\|X\|_{\mathbb{C}^{n \times n}} \leq 1\right\}$ is the closed unit ball in $\mathbb{C}^{n \times n}$. Moreover, the solution $X$ is given by the convergent series

$$
X=\sum_{k=0}^{\infty} B_{k},
$$


where

$$
B_{0}=B, \quad B_{k+1}=B_{k} A B_{k}+B, \quad k \in \mathbb{N}_{0} .
$$

Proof. Introducing the map

$$
F: \mathbb{C}^{n \times n} \longrightarrow \mathbb{C}^{n \times n}, \quad F(X)=X A X+B,
$$

the estimate

$$
\|F(X)\|_{\mathbb{C}^{n \times n}}=\|B+X A X\|_{\mathbb{C}^{n \times n}} \leq\|B\|_{\mathbb{C}^{n \times n}}+\|X\|_{\mathbb{C}^{n \times n}}^{2}\|A\|_{\mathbb{C}^{n \times n}}<1, \quad X \in \bar{K},
$$

combined with (3.42) shows that $F$ maps $\bar{K}$ into itself. Moreover, using

$$
\begin{aligned}
& \|F(X)-F(Y)\|_{\mathbb{C}^{n \times n}}=\|X A X-Y A Y\|_{\mathbb{C}^{n \times n}} \\
& =\|X A X-X A Y+X A Y-Y A Y\|_{\mathbb{C}^{n \times n}} \\
& \leq\left(\|X\|_{\mathbb{C}^{n \times n}}+\|Y\|_{\mathbb{C}^{n \times n}}\right)\|A\|_{\mathbb{C}^{n \times n}}\|X-Y\|_{\mathbb{C}^{n \times n}} \\
& \leq 2\|A\|_{\mathbb{C}^{n \times n}}\|X-Y\|_{\mathbb{C}^{n \times n}}, \quad X, Y \in \bar{K},
\end{aligned}
$$

(3.42) implies that $F$ is a strict contraction. An application of Banach's fixed point theorem then proves the existence of a unique fixed point $X \in \bar{K}$. Moreover, the fixed point $X$ can be obtained as the limit of the sequence $\left\{B_{k}\right\}_{k \in \mathbb{N}}$, where

$$
B_{k+1}=F\left(B_{k}\right), \quad k \in \mathbb{N}_{0},
$$

and any fixed $B_{0} \in \bar{K}$,

$$
X=\lim _{k \rightarrow \infty} F\left(B_{k}\right)
$$

Taking $B_{0}=B$ proves representations (3.44) and (3.45), which in turn completes the proof since any fixed point of $F$ is a solution of the matrix-valued Riccati equation (3.43).

Corollary 3.6. Assume $A_{j}, B_{j}, j=1,2$, satisfy the assumptions of Lemma 3.5 and $X_{j}, j=1,2$, are the corresponding (unique) solutions of the matrix-valued Riccati equations

$$
Y=Y A_{j} Y+B_{j}, \quad j=1,2
$$

in the closed unit ball $\bar{K}$. Then

$$
\left\|X_{1}-X_{2}\right\|_{\mathbb{C}^{n \times n}} \leq \frac{\left\|A_{1}-A_{2}\right\|_{\mathbb{C}^{n \times n}}+\left\|B_{1}-B_{2}\right\|_{\mathbb{C}^{n \times n}}}{1-2 \min _{j=1,2}\left\|A_{j}\right\|_{\mathbb{C}^{n \times n}}} .
$$

Proof. For $X_{j} \in \bar{K}, j=1,2$ the estimate

$$
\begin{aligned}
\| & X_{1}-X_{2}\left\|_{\mathbb{C}^{n \times n}}=\right\| B_{1}-B_{2}+X_{1} A_{1} X_{1}-X_{2} A_{2} X_{2} \|_{\mathbb{C}^{n \times n}} \\
\leq & \left\|B_{1}-B_{2}\right\|_{\mathbb{C}^{n \times n}}+\left\|X_{1} A_{1} X_{1}-X_{2} A_{2} X_{2}\right\|_{\mathbb{C}^{n \times n}} \\
= & \left\|B_{1}-B_{2}\right\|_{\mathbb{C}^{n \times n}}+\left\|X_{1} A_{1} X_{1}-X_{2} A_{1} X_{2}\right\|_{\mathbb{C}^{n \times n}} \\
& +\left\|X_{2} A_{1} X_{2}-X_{2} A_{2} X_{2}\right\|_{\mathbb{C}^{n \times n}} \\
\leq & \left\|B_{1}-B_{2}\right\|_{\mathbb{C}^{n \times n}}+2\left\|A_{1}\right\|_{\mathbb{C}^{n \times n}}\left\|X_{1}-X_{2}\right\|_{\mathbb{C}^{n \times n}} \\
& +\left\|X_{2}\right\|_{\mathbb{C}^{n \times n}}^{2}\left\|A_{1}-A_{2}\right\|_{\mathbb{C}^{n \times n}} \\
\leq & \left\|A_{1}-A_{2}\right\|_{\mathbb{C}^{n \times n}}+\left\|B_{1}-B_{2}\right\|_{\mathbb{C}^{n \times n}}+2\left\|A_{1}\right\|_{\mathbb{C}^{n \times n}}\left\|X_{1}-X_{2}\right\|_{\mathbb{C}^{n \times n}}
\end{aligned}
$$


shows that

$$
\left\|X_{1}-X_{2}\right\|_{\mathbb{C}^{n \times n}} \leq \frac{\left\|A_{1}-A_{2}\right\|_{\mathbb{C}^{n \times n}}+\left\|B_{1}-B_{2}\right\|_{\mathbb{C}^{n \times n}}}{1-2\left\|A_{1}\right\|_{\mathbb{C}^{n \times n}}}
$$

Analogously, one proves that

$$
\left\|X_{1}-X_{2}\right\|_{\mathbb{C}^{n \times n}} \leq \frac{\left\|A_{1}-A_{2}\right\|_{\mathbb{C}^{n \times n}}+\left\|B_{1}-B_{2}\right\|_{\mathbb{C}^{n \times n}}}{1-2\left\|A_{2}\right\|_{\mathbb{C}^{n \times n}}} .
$$

Combining (3.54) and (3.55) yields (3.52).

After these preparations we are able to prove the following fundamental uniqueness result.

Theorem 3.7. Assume Hypothesis 2.7 and let $x_{0} \in \mathbb{R}$.

(i) In the case of a matrix-valued Schrödinger operator $H, g\left(z, x_{0}\right)$ and $g^{\prime}\left(z, x_{0}\right)$ for all $z \in \mathbb{C}_{+}$, uniquely determine the matrix-valued $m \times m$ potential $Q(x)$ for a.e. $x \in \mathbb{R}$.

(ii) In the case of a Dirac-type operator $D$ assume $x_{0}$ is a point of Lebesgue continuity of $B$ and define $g^{\prime}\left(z, x_{0}\right)$ by the right-hand side of (3.29). Then $g\left(z, x_{0}\right)$, $g^{\prime}\left(z, x_{0}\right)$ for all $z \in \mathbb{C}_{+}$, and the $m \times m$ coefficients $B_{1,1}\left(x_{0}\right), B_{1,2}\left(x_{0}\right)$ uniquely determine the $2 m \times 2 m$ matrix $B(x)=\left(\begin{array}{cc}B_{1,1}(x) & B_{1,2}(x) \\ B_{1,2}(x) & -B_{1,1}(x)\end{array}\right)$ for a.e. $x \in \mathbb{R}$.

Proof. Since $M\left(z, x_{0}\right)$ uniquely determines $T$ (i.e., $H$ or $D$ ) by Theorem 2.12, it suffices to show that $g\left(z, x_{0}\right)$ and $g^{\prime}\left(z, x_{0}\right)$ uniquely determine $M\left(z, x_{0}\right)$ in case (i) and similarly, $g\left(z, x_{0}\right), g^{\prime}\left(z, x_{0}\right)$, and $B_{1,1}\left(x_{0}\right), B_{1,2}\left(x_{0}\right)$ uniquely determine $M\left(z, x_{0}\right)$ in case (ii). We start with the Schrödinger case (i). By (2.36), (3.5), and (3.7),

$$
\operatorname{Im}\left(g\left(z, x_{0}\right)\right)>0 \text { for all } z \in \mathbb{C}_{+}
$$

and hence

$$
\operatorname{spec}\left(g\left(z, x_{0}\right)\right) \cap \operatorname{spec}\left(-g\left(z, x_{0}\right)\right)=\emptyset \text { for all } z \in \mathbb{C}_{+}
$$

by $(3.36)$. Applying Lemma 3.3 (with $A=-B=g\left(z, x_{0}\right)$ and $C=g^{\prime}\left(z, x_{0}\right) \mp I_{n}$ ) to (3.28) (with $\left.x=x_{0}\right)$ then shows that $M_{ \pm}\left(z, x_{0}\right)$ are uniquely determined by $g\left(z, x_{0}\right)$ and $g^{\prime}\left(z, x_{0}\right)$.

In the corresponding Dirac case (ii) one rewrites (3.29) (with $x=x_{0}$ ) in the form

$$
\begin{aligned}
& g\left(z, x_{0}\right)^{-1}\left[I_{m}-z^{-1} B_{1,1}\left(x_{0}\right)\right] M_{+}\left(z, x_{0}\right)+M_{+}\left(z, x_{0}\right)\left[I_{m}-z^{-1} B_{1,1}\left(x_{0}\right)\right] g\left(z, x_{0}\right)^{-1} \\
& =z^{-1}\left[g\left(z, x_{0}\right)^{-1} g^{\prime}\left(z, x_{0}\right) g\left(z, x_{0}\right)^{-1}-B_{1,2}\left(x_{0}\right) g\left(z, x_{0}\right)^{-1}-g\left(z, x_{0}\right)^{-1} B_{1,2}\left(x_{0}\right)\right. \\
& \left.\quad-z g\left(z, x_{0}\right)^{-2}+g\left(z, x_{0}\right)^{-1} B_{1,1}\left(x_{0}\right) g\left(z, x_{0}\right)^{-1}\right] .
\end{aligned}
$$

Thus, for $\operatorname{Im}(z)>0$ sufficiently large, we can again apply Lemma 3.3 (with $A=$ $g\left(z, x_{0}\right)^{-1}\left[I_{m}-z^{-1} B_{1,1}\left(x_{0}\right)\right], B=-\left[I_{m}-z^{-1} B_{1,1}\left(x_{0}\right)\right] g\left(z, x_{0}\right)^{-1}$, and $C=$ the r.-h.s. of (3.58)) since clearly

$$
\operatorname{spec}\left(g\left(z, x_{0}\right)^{-1}\left[I_{m}-z^{-1} B_{1,1}\left(x_{0}\right)\right]\right) \cap \operatorname{spec}\left(-\left[I_{m}-z^{-1} B_{1,1}\left(x_{0}\right)\right] g\left(z, x_{0}\right)^{-1}\right)=\emptyset
$$

for $\operatorname{Im}(z)>0$ sufficiently large. Hence $M_{+}\left(z, x_{0}\right)$ is uniquely determined for $\operatorname{Im}(z)>0$ sufficiently large. Since $M_{+}\left(\cdot, x_{0}\right)$ is analytic in $\mathbb{C}_{+}$, one concludes that $M_{+}\left(z, x_{0}\right)$ is uniquely determined for all $z \in \mathbb{C}_{+}$. Since $g\left(z, x_{0}\right)=\left[M_{-}\left(z, x_{0}\right)-\right.$ $\left.M_{+}\left(z, x_{0}\right)\right]^{-1}$ is known by hypothesis, this also uniquely determines $M_{-}\left(z, x_{0}\right)$ for all $z \in \mathbb{C}_{+}$.

In either case an application of Theorem 2.12 completes the proof. 
Since $g\left(z, x_{0}\right)$ and $g^{\prime}\left(z, x_{0}\right)$ are analytic in $\mathbb{C}_{+}$, it suffices of course in Theorem 3.7 to know them for an infinite set of $z \in \mathbb{C}_{+}$with an accumulation point in $\mathbb{C}_{+}$.

In the special scalar case $m=1$, the uniqueness result stated in Theorem 3.7 is well-known for one-dimensional Schrödinger operators on $\mathbb{R}$ and can be inferred, for instance, from the results in [76], [79], 80], 31] (it is also explicitly stated in 58). The present matrix extension with $m>1$, however, appears to be new. To the best of our knowledge, the corresponding Dirac case has not previously been studied in the literature from this uniqueness point of view.

Next we turn to a local version of Theorem 3.7, our principal new result.

Theorem 3.8. Suppose $B_{j}, j=1,2$ satisfy Hypothesis 2.9 and let $a>0$ and $x_{0} \in$ $\mathbb{R}$. Introduce $g_{j}\left(z, x_{0}\right)=\left[M_{j,-}\left(z, x_{0}\right)-M_{j,+}\left(z, x_{0}\right)\right]^{-1}$, where $M_{j, \pm}\left(z, x_{0}\right), j=1,2$ denote the $m \times m$ Weyl-Titchmarsh matrices associated with the corresponding Hamiltonian systems (2.4) on $\left(x_{0}, \pm \infty\right)$.

(i) In the case of matrix-valued Schrödinger operators $H_{j}, j=1,2$ suppose that

$$
\left\|g_{1}\left(z, x_{0}\right)-g_{2}\left(z, x_{0}\right)\right\|_{\mathbb{C}^{m \times m}}+\left\|g_{1}^{\prime}\left(z, x_{0}\right)-g_{2}^{\prime}\left(z, x_{0}\right)\right\|_{\mathbb{C}^{m \times m}} \underset{|z| \rightarrow \infty}{=} O\left(e^{-2 \operatorname{Im}\left(z^{1 / 2}\right) a}\right)
$$

along a ray with $0<\arg (z)<\pi$. Then

$$
Q_{1}(x)=Q_{2}(x) \text { for a.e. } x \in\left[x_{0}-a, x_{0}+a\right] .
$$

(ii) In the case of Dirac-type operators assume that $x_{0}$ is a point of Lebesgue continuity of $B_{j}$ and define $g_{j}^{\prime}\left(z, x_{0}\right), j=1,2$, by the right-hand side of (3.29). Moreover, suppose that

$$
\left\|g_{1}\left(z, x_{0}\right)-g_{2}\left(z, x_{0}\right)\right\|_{\mathbb{C}^{m \times m}}+\left\|g_{1}^{\prime}\left(z, x_{0}\right)-g_{2}^{\prime}\left(z, x_{0}\right)\right\|_{\mathbb{C}^{m \times m}} \underset{|z| \rightarrow \infty}{=} O\left(e^{-2 \operatorname{Im}(z) a}\right)
$$

along a ray with $0<\arg (z)<\pi / 2$ and along a ray with $\pi / 2<\arg (z)<\pi$. In addition, assume that

$$
\left(B_{1}\left(x_{0}\right)\right)_{1,1}=\left(B_{2}\left(x_{0}\right)\right)_{1,1}, \quad\left(B_{1}\left(x_{0}\right)\right)_{1,2}=\left(B_{2}\left(x_{0}\right)\right)_{1,2} .
$$

Then

$$
B_{1}(x)=B_{2}(x) \text { for a.e. } x \in\left[x_{0}-a, x_{0}+a\right] .
$$

Proof. In the Schrödinger context one observes from Lemmas 3.3 and 3.4, and (3.28) (for $x=x_{0}$ ) that

$$
\begin{aligned}
& M_{1, \pm}\left(z, x_{0}\right)-M_{2, \pm}\left(z, x_{0}\right) \\
& =(2 \pi i)^{-1} \oint_{\Gamma_{1,2}} d \zeta\left[\left(g_{1}\left(z, x_{0}\right)-\zeta\right)^{-1}\left[g_{1}^{\prime}\left(z, x_{0}\right) \mp I_{m}\right]\left(-g_{1}\left(z, x_{0}\right)-\zeta\right)^{-1}\right. \\
& \left.\quad-\left(g_{2}\left(z, x_{0}\right)-\zeta\right)^{-1}\left[g_{2}^{\prime}\left(z, x_{0}\right) \mp I_{m}\right]\left(-g_{2}\left(z, x_{0}\right)-\zeta\right)^{-1}\right] .
\end{aligned}
$$

Here $\Gamma_{1,2}$ is a closed and counterclockwise oriented Jordan contour which encircles $\operatorname{spec}\left(g_{1}\left(z, x_{0}\right)\right) \cup \operatorname{spec}\left(g_{2}\left(z, x_{0}\right)\right)$ with winding number +1 and $\operatorname{spec}\left(-g_{1}\left(z, x_{0}\right)\right) \cup$ $\operatorname{spec}\left(-g_{2}\left(z, x_{0}\right)\right)$ with winding number 0 , and whose existence is guaranteed by Lemma 3.4 (cf. (3.57)). Using the asymptotic expansion (cf. [36])

$$
g_{j}\left(z, x_{0}\right) \underset{|z| \rightarrow \infty}{=}(i / 2) z^{-1 / 2} I_{m}+o\left(z^{-1}\right), \quad 0<\arg (z)<\pi, j=1,2,
$$


(3.60) and a standard $\varepsilon / 3$-argument yield

$$
\left\|M_{1, \pm}\left(z, x_{0}\right)-M_{2, \pm}\left(z, x_{0}\right)\right\|_{\mathbb{C}^{m \times m}} \underset{|z| \rightarrow \infty}{=} O\left(e^{-2 \operatorname{Im}\left(z^{1 / 2}\right) a}\right)
$$

along a ray with $\arg (z)=\pi-\varepsilon$. Hence one obtains (3.61) by Theorem 2.13 (i).

In the case of Dirac-type operators one infers from (3.58) that

$$
\begin{aligned}
& g_{j}\left(z, x_{0}\right)^{-1}\left[I_{m}-z^{-1}\left(B_{j}\left(x_{0}\right)\right)_{1,1}\right] M_{j,+}\left(z, x_{0}\right) \\
& \quad+M_{j,+}\left(z, x_{0}\right)\left[I_{m}-z^{-1}\left(B_{j}\left(x_{0}\right)\right)_{1,1}\right] g_{j}\left(z, x_{0}\right)^{-1} \\
& =z^{-1}\left[g_{j}\left(z, x_{0}\right)^{-1} g_{j}^{\prime}\left(z, x_{0}\right) g_{j}\left(z, x_{0}\right)^{-1}-\left(B_{j}\left(x_{0}\right)\right)_{1,2} g_{j}\left(z, x_{0}\right)^{-1}\right. \\
& \quad-g_{j}\left(z, x_{0}\right)^{-1}\left(B_{j}\left(x_{0}\right)\right)_{1,2}-z g_{j}\left(z, x_{0}\right)^{-2}+g_{j}\left(z, x_{0}\right)^{-1}\left(B_{j}\left(x_{0}\right)\right)_{1,1} g_{j}\left(z, x_{0}\right)^{-1} \\
& j=1,2 \quad(3.68
\end{aligned}
$$

and then proceeds in exactly the same manner as in (3.65)-(3.67) replacing (3.66) by (cf. [37)

$$
g_{j}\left(z, x_{0}\right) \underset{|z| \rightarrow \infty}{=}(i / 2) I_{m}+o(1), \quad 0<\arg (z)<\pi, j=1,2 .
$$

For an alternative proof of (3.67) one can multiply (3.28) (for $\left.x=x_{0}\right)$ and $(3.68)$ by $(-i)$ and directly apply Lemma 3.3 (ii) taking into account $(3.66)$ and (3.69), respectively.

As mentioned in [60], the ray with $0<\arg (z)<\pi$ in Theorem 3.8 (i) is of no particular significance and can be replaced by any non-selfintersecting Jordan arc that tends to infinity in the sector $\varepsilon \leq \arg (z) \leq \pi-\varepsilon$ for some $0<\varepsilon<\pi / 2$. The analogous comment applies to Theorem 3.8 (ii)

As discussed in Marchenko's monograph [106, p. 193-195], it is possible to reduce certain Dirac operators $D$ with potentials $B(x)$ of the type 2.10 ) to $2 m \times 2 m$ matrixvalued Schrödinger operators by essentially taking the square of the Dirac operator D. This permits one to relate the $M$-functions of the $2 m \times 2 m$ Schrödinger and Dirac-type operators and we next explore this connection in some detail. In order to indicate that $B$ is temporarily of the special form $(2.10)$, we denote it by $\widetilde{B}$ in the following and assume in addition to Hypothesis 2.2 that

$$
\widetilde{B}=\left(\begin{array}{cc}
\widetilde{B}_{1,1} & \widetilde{B}_{1,2} \\
\widetilde{B}_{1,2} & -\widetilde{B}_{1,1}
\end{array}\right) \in C^{1}(\mathbb{R})^{2 m \times 2 m}
$$

(this can be improved but is of little relevance in our context). Denoting the resulting Dirac operator by $\widetilde{D}$,

$$
\widetilde{D}=J \frac{d}{d x}-\widetilde{B},
$$

one computes, using $J^{2}=-I_{2 m}$ and $J \widetilde{B}(x)+\widetilde{B}(x) J=0$,

$$
\widetilde{D}^{2}=-I_{2 m} \frac{d^{2}}{d x^{2}}+\widetilde{B}^{2}-J \widetilde{B}^{\prime}
$$

and hence obtains the $2 m \times 2 m$ matrix-valued Schrödinger operator

$$
\widetilde{H}=-I_{2 m} \frac{d^{2}}{d x^{2}}+\widetilde{Q},
$$


where

$$
\begin{aligned}
\widetilde{Q}(x) & =\widetilde{B}(x)^{2}-J \widetilde{B}^{\prime}(x) \\
& =\left(\begin{array}{cc}
\widetilde{B}_{1,1}(x)^{2}+\widetilde{B}_{1,2}(x)^{2}+\widetilde{B}_{1,2}^{\prime}(x) & \widetilde{B}_{1,1}(x) \widetilde{B}_{1,2}(x)-\widetilde{B}_{1,2}(x) \widetilde{B}_{1,1}(x)-\widetilde{B}_{1,1}^{\prime}(x) \\
\widetilde{B}_{1,2}(x) \widetilde{B}_{1,1}(x)-\widetilde{B}_{1,1}(x) \widetilde{B}_{1,2}(x)-\widetilde{B}_{1,1}^{\prime}(x) & \widetilde{B}_{1,1}(x)^{2}+\widetilde{B}_{1,2}(x)^{2}-\widetilde{B}_{1,2}^{\prime}(x)
\end{array}\right) .
\end{aligned}
$$

Since

$$
\left(\widetilde{H}-z^{2}\right)=(\widetilde{D}-z)(\widetilde{D}+z),
$$

linearly independent solutions $\Phi_{+}\left(z^{2}, x, x_{0}\right), \Phi_{-}\left(z^{2}, x, x_{0}\right)$ of $\left(\widetilde{H}-z^{2}\right) \Phi\left(z^{2}, x, x_{0}\right)=$ 0 are of the type

$$
\Phi_{ \pm}\left(z^{2}, x, x_{0}\right)=\left(\begin{array}{ll}
\psi_{ \pm, 1}\left(z, x, x_{0}\right) & \psi_{ \pm, 1}\left(-z, x, x_{0}\right) \\
\psi_{ \pm, 2}\left(z, x, x_{0}\right) & \psi_{ \pm, 2}\left(-z, x, x_{0}\right)
\end{array}\right), \quad z^{2} \in \mathbb{C}_{+}, x, x_{0} \in \mathbb{R},
$$

where, in accordance with (2.44), the $m$ columns of $\Psi_{ \pm}\left(z, x, x_{0}\right)$

$$
\begin{aligned}
\Psi_{ \pm}\left(z, x, x_{0}\right) & =\left(\begin{array}{l}
\psi_{ \pm, 1}\left(z, x, x_{0}\right) \\
\psi_{ \pm, 2}\left(z, x, x_{0}\right)
\end{array}\right) \\
& =\left(\begin{array}{ll}
\theta_{1}\left(z, x, x_{0}\right) & \phi_{1}\left(z, x, x_{0}\right) \\
\theta_{2}\left(z, x, x_{0}\right) & \phi_{2}\left(z, x, x_{0}\right)
\end{array}\right)\left(\begin{array}{c}
I_{m} \\
M_{ \pm}^{\widetilde{D}}\left(z, x_{0}\right)
\end{array}\right), \quad z \in \mathbb{C} \backslash \mathbb{R}, x, x_{0} \in \mathbb{R}
\end{aligned}
$$

form a basis for the nullspace of $(\widetilde{D}-z)$ Here, in obvious notation, $M_{ \pm}^{\widetilde{D}}\left(z, x_{0}\right)$ abbreviates the corresponding $m \times m$ half-line Weyl-Titchmarsh matrices associated with $\widetilde{D}$, given by

$$
\begin{aligned}
M_{ \pm}^{\widetilde{D}}\left(z, x_{0}\right) & =\psi_{ \pm, 2}\left(z, x_{0}, x_{0}\right) \psi_{ \pm, 1}\left(z, x_{0}, x_{0}\right)^{-1}=\psi_{ \pm, 2}\left(z, x_{0}, x_{0}\right), \\
M_{ \pm}^{\widetilde{D}}\left(-z, x_{0}\right) & =M_{ \pm}^{\widetilde{D}}\left(-\bar{z}, x_{0}\right)^{*}, \quad z \in \mathbb{C}_{+},
\end{aligned}
$$

using the normalization $\psi_{ \pm, j}\left(z, x_{0}, x_{0}\right)=I_{m}, j=1,2$. The corresponding $2 m \times$ $2 m$ half-line Weyl-Titchmarsh matrices $M_{ \pm}^{\widetilde{H}}\left(z^{2}, x_{0}\right)$, associated with $\widetilde{H}$, are then defined by

$$
M_{ \pm}^{\widetilde{H}}\left(z^{2}, x_{0}\right)=\Phi_{ \pm}\left(z^{2}, x_{0}, x_{0}\right) \Phi_{ \pm}\left(z^{2}, x_{0}, x_{0}\right)^{-1}, \quad z^{2} \in \mathbb{C}_{+} .
$$

Next we explicitly compute $M_{ \pm}^{\widetilde{H}}\left(z^{2}, x_{0}\right)$ in terms of $M_{ \pm}^{\widetilde{D}}\left(z, x_{0}\right), M_{ \pm}^{\widetilde{D}}\left(-z, x_{0}\right)$.

Lemma 3.9. Assume Hypothesis 2.9, (3.70), and $\arg (z) \in(0, \pi / 2)$. Then

$$
\begin{aligned}
M_{ \pm}^{\widetilde{H}}\left(z^{2}, x_{0}\right)= & \left(M_{ \pm}^{\widetilde{H}}\left(z, x_{0}\right)_{j, k}\right)_{1 \leq j, k \leq 2} \\
M_{ \pm}^{\widetilde{H}}\left(z^{2}, x_{0}\right)_{1,1}= & \widetilde{B}_{1,2}\left(x_{0}\right) \\
& +2 z M_{ \pm}^{\widetilde{D}}\left(z, x_{0}\right)\left[M_{ \pm}^{\widetilde{D}}\left(-z, x_{0}\right)-M_{ \pm}^{\widetilde{D}}\left(z, x_{0}\right)\right]^{-1} M_{ \pm}^{\widetilde{D}}\left(-z, x_{0}\right) \\
M_{ \pm}^{\widetilde{H}}\left(z^{2}, x_{0}\right)_{1,2}= & -\widetilde{B}_{1,1}\left(x_{0}\right) \\
& -z\left[M_{ \pm}^{\widetilde{D}}\left(-z, x_{0}\right)+M_{ \pm}^{\widetilde{D}}\left(z, x_{0}\right)\right]\left[M_{ \pm}^{\widetilde{D}}\left(-z, x_{0}\right)-M_{ \pm}^{\widetilde{D}}\left(z, x_{0}\right)\right]^{-1} \\
M_{ \pm}^{\widetilde{H}}\left(z^{2}, x_{0}\right)_{2,1}= & -\widetilde{B}_{1,1}\left(x_{0}\right) \\
& -z\left[M_{ \pm}^{\widetilde{D}}\left(-z, x_{0}\right)+M_{ \pm}^{\widetilde{D}}\left(z, x_{0}\right)\right]^{-1}\left[M_{ \pm}^{\widetilde{D}}\left(-z, x_{0}\right)-M_{ \pm}^{\widetilde{D}}\left(z, x_{0}\right)\right] \\
M_{ \pm}^{\widetilde{H}}\left(z^{2}, x_{0}\right)_{2,2}= & -\widetilde{B}_{1,2}\left(x_{0}\right)+2 z\left[M_{ \pm}^{\widetilde{D}}\left(-z, x_{0}\right)-M_{ \pm}^{\widetilde{D}}\left(z, x_{0}\right)\right]^{-1} .
\end{aligned}
$$


Proof. Combining (3.79),

$$
\begin{aligned}
& \Phi_{ \pm}\left(z^{2}, x_{0}, x_{0}\right)=\left(\begin{array}{cc}
I_{m} & I_{m} \\
M_{ \pm}^{\tilde{D}}\left(z, x_{0}\right) & M_{ \pm}^{\widetilde{D}}\left(-z, x_{0}\right)
\end{array}\right) \\
& \Phi_{ \pm}\left(z^{2}, x_{0}, x_{0}\right)^{-1} \\
& =\left(\begin{array}{cc}
{\left[M_{ \pm}^{\widetilde{D}}\left(-z, x_{0}\right)-M_{ \pm}^{\widetilde{D}}\left(z, x_{0}\right)\right] M_{ \pm}^{\widetilde{D}}\left(-z, x_{0}\right)} & -\left[M_{ \pm}^{\widetilde{D}}\left(-z, x_{0}\right)-M_{ \pm}^{\widetilde{D}}\left(z, x_{0}\right)\right]^{-1} \\
-\left[M_{ \pm}^{\widetilde{D}}\left(-z, x_{0}\right)-M_{ \pm}^{\widetilde{D}}\left(z, x_{0}\right)\right] M_{ \pm}^{\widetilde{D}}\left(z, x_{0}\right) & {\left[M_{ \pm}^{\widetilde{D}}\left(-z, x_{0}\right)-M_{ \pm}^{\tilde{D}}\left(z, x_{0}\right)\right]^{-1}}
\end{array}\right) \\
& J \Psi_{ \pm}^{\prime}\left(z, x_{0}, x_{0}\right)=\left(\begin{array}{c}
\widetilde{B}_{1,1}\left(x_{0}\right)+z I_{m}+\widetilde{B}_{1,2}\left(x_{0}\right) M_{ \pm}^{\widetilde{D}}\left(z, x_{0}\right) \\
\widetilde{B}_{1,2}\left(x_{0}\right)-\widetilde{B}_{1,1}\left(x_{0}\right) M_{ \pm}^{\widetilde{D}}\left(z, x_{0}\right)+z M_{ \pm}^{\widetilde{D}}\left(z, x_{0}\right)
\end{array}\right)
\end{aligned}
$$

yields (3.80) after straightforward matrix computations.

\section{Matrix-Valued Jacobi Operators}

In this section we prove analogous uniqueness theorems for matrix-valued Jacobi (i.e., second-order finite difference) operators. We could consider analogous Diractype difference operators and hence introduce discrete Hamiltonian systems as in Section 2, but for simplicity we will confine ourselves to the Jacobi case.

As the basic hypothesis of this section we adopt the following.

Hypothesis 4.1. Let $m \in \mathbb{N}$ and consider the sequences of self-adjoint $m \times m$ matrices

$$
\begin{array}{ll}
A=\{A(k)\}_{k \in \mathbb{Z}}, & A(k)=A(k)^{*}, A(k)>0, k \in \mathbb{Z}, \\
B=\{B(k)\}_{k \in \mathbb{Z}}, & B(k)=B(k)^{*}, k \in \mathbb{Z} .
\end{array}
$$

Moreover, we assume that $A(k)$ and $B(k)$ are uniformly bounded with respect to $k$, that is, there exists a $C>0$, such that

$$
\|A(k)\|_{\mathbb{C}^{m \times m}}+\|B(k)\|_{\mathbb{C}^{m \times m}} \leq C, \quad k \in \mathbb{Z} .
$$

Next, denote by $S^{ \pm}$the shift operators in $\ell^{\infty}(\mathbb{Z})^{m}$ and $\ell^{\infty}(\mathbb{Z})^{m \times m}$, that is,

$$
\left(S^{ \pm} g\right)(k)=g^{ \pm}(k)=g(k \pm 1) \text { for } g \in \ell^{\infty}(\mathbb{Z})^{m} \text { or } g \in \ell^{\infty}(\mathbb{Z})^{m \times m}, k \in \mathbb{Z} .
$$

Given Hypothesis 4.1, the matrix-valued Jacobi operator $H$ in $\ell^{2}(\mathbb{Z})^{m}$ is then defined by

$$
H=A S^{+}+A^{-} S^{-}+B, \quad \operatorname{dom}(H)=\ell^{2}(\mathbb{Z})^{m} .
$$

Because of hypothesis (4.2), $H$ is a bounded symmetric operator and hence selfadjoint. In particular, the difference expression $A S^{+}+A^{-} S^{-}+B$ induced by (4.4) is in the limit point case at $\pm \infty$. We chose to adopt (4.2) for simplicity only. Our formalism extends to unbounded Jacobi operators and to cases where the difference expression associated with (4.4) is in the limit circle case at $+\infty$ and/or $-\infty$. We just note in passing that without assuming (4.2), the difference expression $A S^{+}+A^{-} S^{-}+B$ is in the limit point case at $\pm \infty$ if $\sum_{k=k_{0}}^{ \pm \infty}\|A(k)\|_{\mathbb{C}^{m \times m}}^{-1}=\infty$ (see, e.g., 19, Theorem VII.2.9]).

The Green's matrix associated with $H$ will be denoted by $G(z, k, \ell)$ in the following,

$$
G(z, k, \ell)=(H-z)^{-1}(k, \ell), \quad z \in \mathbb{C} \backslash \mathbb{R}, k, \ell \in \mathbb{Z} .
$$


Next, fix a site $k_{0} \in \mathbb{Z}$ and in analogy to 2.13)-(2.15) define $m \times m$ matrix-valued solutions $\phi\left(z, k, k_{0}\right)$ and $\theta\left(z, k, k_{0}\right)$ of the equation

$$
A(k) \psi(z, k+1)+A(k-1) \psi(z, k-1)+\left(B(k)-z I_{m}\right) \psi(z, k)=0, \quad z \in \mathbb{C}, k \in \mathbb{Z},
$$

satisfying the initial conditions

$$
\theta\left(z, k_{0}, k_{0}\right)=\phi\left(z, k_{0}+1, k_{0}\right)=I_{m}, \quad \phi\left(z, k_{0}, k_{0}\right)=\theta\left(z, k_{0}+1, k_{0}\right)=0 .
$$

As in 2.44, (2.45) one then introduces $m \times m$ matrix-valued Weyl solutions $\psi_{ \pm}\left(z, k, k_{0}\right)$ associated with $H$ defined by

$$
\psi_{ \pm}\left(z, k, k_{0}\right)=\theta\left(z, k, k_{0}\right)-\phi\left(z, k, k_{0}\right) A\left(k_{0}\right)^{-1} M_{ \pm}\left(z, k_{0}\right), \quad z \in \mathbb{C} \backslash \mathbb{R}, k \in \mathbb{Z},
$$

with the properties

$$
\begin{aligned}
& A(k) \psi_{ \pm}\left(z, k+1, k_{0}\right)+A(k-1) \psi_{ \pm}\left(z, k-1, k_{0}\right)+(B(k)-z) \psi_{ \pm}\left(z, k, k_{0}\right)=0 \\
& \psi_{ \pm}\left(z, \cdot, k_{0}\right) \in \ell^{2}\left(\left(k_{0}, \pm \infty\right) \cap \mathbb{Z}\right)^{m \times m}, \quad z \in \mathbb{R}, k \in \mathbb{Z},
\end{aligned}
$$

where $M_{ \pm}\left(z, k_{0}\right)$ denote the Weyl-Titchmarsh matrices associated with $H$. Since by assumption $A S^{+}+A^{-} S^{-}+B$ is in the limit point case at $\pm \infty, M_{ \pm}\left(z, k_{0}\right)$ in (4.8) are uniquely determined by the requirement (4.10). We also note that by a standard argument,

$$
\operatorname{det}\left(\phi\left(z, k, k_{0}\right)\right) \neq 0 \text { for all } k \in \mathbb{Z} \backslash\left\{k_{0}\right\} \text { and } z \in \mathbb{C} \backslash \mathbb{R},
$$

since otherwise one could construct a Dirichlet-type eigenvalue $z \in \mathbb{C} \backslash \mathbb{R}$ for $H$ restricted to the finite segment $k_{0}+1, \ldots, k-1$ for $k \geq k_{0}+1$ of $\mathbb{Z}$ (and similarly for $\left.k \leq k_{0}-1\right)$. Thus, introducing

$$
M_{N}\left(z, k_{0}\right)=-\phi\left(z, N, k_{0}\right)^{-1} \theta\left(z, N, k_{0}\right), \quad z \in \mathbb{C} \backslash \mathbb{R}, N \in \mathbb{Z} \backslash\left\{k_{0}\right\},
$$

one can then compute $M_{ \pm}\left(z, k_{0}\right)$ by the limiting relation

$$
M_{ \pm}\left(z, k_{0}\right)=\lim _{N \rightarrow \pm \infty} M_{N}\left(z, k_{0}\right), \quad z \in \mathbb{C} \backslash \mathbb{R}
$$

the limit being unique since $A S^{+}+A^{-} S^{-}+B$ is in the limit point case at $\pm \infty$. Alternatively, 4.8 yields

$$
M_{ \pm}\left(z, k_{0}\right)=-A\left(k_{0}\right) \psi_{ \pm}\left(z, k_{0}+1, k_{0}\right), \quad z \in \mathbb{C} \backslash \mathbb{R} .
$$

More generally, recalling

$$
\operatorname{det}\left(\psi_{ \pm}\left(z, k, k_{0}\right)\right) \neq 0 \text { for all } k \in \mathbb{Z} \text { and } z \in \mathbb{C} \backslash \mathbb{R}
$$

by an argument analogous to that following (4.11), we now introduce

$$
M_{ \pm}(z, k)=-A(k) \psi_{ \pm}\left(z, k+1, k_{0}\right) \psi_{ \pm}\left(z, k, k_{0}\right)^{-1}, \quad z \in \mathbb{C} \backslash \mathbb{R}, k \in \mathbb{Z}
$$

One easily verifies that $M_{ \pm}(z, k)$ represent the Weyl-Titchmarsh $M$-matrices associated with the reference point $k \in \mathbb{Z}$. Moreover, one obtains the Riccati-type equation for $M_{ \pm}(z, k)$,

$$
M_{ \pm}(z, k)+A(k-1) M_{ \pm}(z, k-1)^{-1} A(k-1)-(B(k)-z)=0, \quad z \in \mathbb{C} \backslash \mathbb{R}, k \in \mathbb{Z}
$$

as a result of (4.9). 
Next, we define the self-adjoint half-line Jacobi operators $H_{ \pm, k_{0}}$ in $\ell^{2}\left(\left(k_{0}, \pm \infty\right) \cap\right.$ $\mathbb{Z})^{m}$ by

$$
H_{ \pm, k_{0}}=\left.P_{ \pm, k_{0}} H P_{ \pm, k_{0}}\right|_{\ell^{2}\left(\left(k_{0}, \pm \infty\right) \cap \mathbb{Z}\right)^{m}}, \quad k_{0} \in \mathbb{Z},
$$

where $P_{ \pm, k_{0}}$ are the orthogonal projections onto the subspaces $\ell^{2}\left(\left[k_{0}, \pm \infty\right) \cap \mathbb{Z}\right)^{m}$. By inspection, $H_{ \pm, k_{0}}$ satisfy Dirichlet boundary conditions at $k_{0} \mp 1$,

$$
\begin{aligned}
\left(H_{+, k_{0}} f\right)\left(k_{0}\right) & =A\left(k_{0}\right) f^{+}\left(k_{0}\right)+B\left(k_{0}\right) f\left(k_{0}\right), \\
\left(H_{-, k_{0}} f\right)\left(k_{0}\right) & =A^{-}\left(k_{0}\right) f^{-}\left(k_{0}\right)+B\left(k_{0}\right) f\left(k_{0}\right), \\
\left(H_{ \pm, k_{0}} f\right)(k) & =A(k) f^{+}(k)+A^{-}(k) f^{-}(k)+B(k) f(k), \quad k \gtreqless k_{0} \pm 1, \\
f \in \operatorname{dom}\left(H_{ \pm, k_{0}}\right) & =\ell^{2}\left(\left(k_{0}, \pm \infty\right) \cap \mathbb{Z}\right)^{m}
\end{aligned}
$$

(i.e., formally, $\left.f\left(k_{0} \mp 1\right)=0\right)$. We also introduce Weyl $m$-matrices $m_{ \pm}\left(z, k_{0}\right)$ associated with $H_{ \pm, k_{0}}$ by

$$
\begin{aligned}
m_{ \pm}\left(z, k_{0}\right) & =Q_{k_{0}}\left(H_{ \pm, k_{0}}-z\right)^{-1} Q_{k_{0}}, \\
& =G_{ \pm, k_{0}}\left(z, k_{0}, k_{0}\right), \quad z \in \mathbb{C} \backslash \mathbb{R}, k_{0} \in \mathbb{Z} .
\end{aligned}
$$

Here $Q_{k_{0}}$ are orthogonal projections onto the $m$-dimensional subspaces $\ell^{2}\left(\left\{k_{0}\right\}\right)^{m}$, $k_{0} \in \mathbb{Z}$ and

$$
G_{ \pm, k_{0}}(z, k, \ell)=\left(H_{ \pm, k_{0}}-z\right)^{-1}(k, \ell), \quad z \in \mathbb{C} \backslash \mathbb{R}, k, \ell \in \mathbb{Z}
$$

represents the Green's matrix of $H_{ \pm, k_{0}}$.

In order to find the connection between $m_{ \pm}\left(z, k_{0}\right)$ and $M_{ \pm}\left(z, k_{0}\right)$ we briefly discuss the Green's matrices $G_{ \pm, k_{0}}(z, k, \ell)$ and $G(z, k, \ell)$ associated with $H_{ \pm, k_{0}}$ and $H$ next.

First we recall the definition of the Wronskian $W(f, g)(k)$ of two sequences of matrices $f(\cdot), g(\cdot) \in \mathbb{C}^{m \times m}$ given by

$$
W(f, g)(k)=f(k) A(k) g(k+1)-f(k+1) A(k) g(k), \quad k \in \mathbb{Z} .
$$

We note that for any two matrix-valued solutions $\varphi(z, \cdot)$ and $\psi(z, \cdot)$ of (4.6) the Wronskian $W\left(\varphi(\bar{z}, \cdot)^{*}, \psi(z, \cdot)\right)(k)$ is independent of $k \in \mathbb{Z}$.

In complete analogy to the scalar Jacobi case (i.e., $m=1$ ) one verifies,

$$
\begin{array}{r}
G_{+, k_{0}}(z, k, \ell)= \begin{cases}-\psi_{+}\left(z, k, k_{0}-1\right) A\left(k_{0}-1\right)^{-1} \phi\left(\bar{z}, \ell, k_{0}-1\right)^{*}, & \ell \leq k, \\
-\phi\left(z, k, k_{0}-1\right) A\left(k_{0}-1\right)^{-1} \psi_{+}\left(\bar{z}, \ell, k_{0}-1\right)^{*}, & \ell \geq k,\end{cases} \\
G_{-, k_{0}}(z, k, \ell)= \begin{cases}\phi\left(z, k, k_{0}+1\right) A\left(k_{0}+1\right)^{-1} \psi_{-}\left(\bar{z}, \ell, k_{0}+1\right)^{*}, & \ell \leq k, \\
\psi_{-}\left(z, k, k_{0}+1\right) A\left(k_{0}+1\right)^{-1} \phi\left(\bar{z}, \ell, k_{0}+1\right)^{*}, & \ell \geq k, \\
z \in \mathbb{C} \backslash \mathbb{R}, & k, \ell \in \mathbb{Z} .\end{cases}
\end{array}
$$

Similarly, using the fact that

$$
\begin{aligned}
& \psi_{+}\left(z, k, k_{0}\right)\left[M_{-}\left(z, k_{0}\right)-M_{+}\left(z, k_{0}\right)\right]^{-1} \psi_{-}\left(\bar{z}, k, k_{0}\right)^{*} \\
& =\psi_{-}\left(z, k, k_{0}\right)\left[M_{-}\left(z, k_{0}\right)-M_{+}\left(z, k_{0}\right)\right]^{-1} \psi_{+}\left(z, k, k_{0}\right) \\
& =\left[M_{-}(z, k)-M_{+}(z, k)\right]^{-1}, \quad k \in \mathbb{Z},
\end{aligned}
$$

and

$$
\begin{aligned}
& A(k) \psi_{+}\left(z, k+1, k_{0}\right)\left[M_{-}\left(z, k_{0}\right)-M_{+}\left(z, k_{0}\right)\right]^{-1} \psi_{-}\left(\bar{z}, k, k_{0}\right)^{*} \\
& -A(k) \psi_{-}\left(z, k+1, k_{0}\right)\left[M_{-}\left(z, k_{0}\right)-M_{+}\left(z, k_{0}\right)\right]^{-1} \psi_{+}\left(\bar{z}, k, k_{0}\right)^{*}=I_{m}, \quad k \in \mathbb{Z},
\end{aligned}
$$


one verifies

$$
G(z, k, \ell)=\left\{\begin{array}{rr}
\psi_{+}\left(z, k, k_{0}\right)\left[M_{-}\left(z, k_{0}\right)-M_{+}\left(z, k_{0}\right)\right]^{-1} \psi_{-}\left(\bar{z}, \ell, k_{0}\right)^{*}, & \ell \leq k, \\
\psi_{-}\left(z, k, k_{0}\right)\left[M_{-}\left(z, k_{0}\right)-M_{+}\left(z, k_{0}\right)\right]^{-1} \psi_{+}\left(\bar{z}, \ell, k_{0}\right)^{*}, & \ell \geq k, \\
z \in \mathbb{C} \backslash \mathbb{R}, & k, \ell \in \mathbb{Z} .
\end{array}\right.
$$

Using (4.7), (4.9), (4.17), and 4.21), one infers that the Weyl-Titchmarsh matrices $M_{ \pm}\left(z, k_{0}\right)$ introduced by (4.13) (resp. (4.14)) and the Weyl $m$-functions $m_{ \pm}\left(z, k_{0}\right)$ defined in (4.20) are related by

$$
M_{+}\left(z, k_{0}\right)=-m_{+}\left(z, k_{0}\right)^{-1}-z I_{m}+B\left(k_{0}\right)
$$

and

$$
M_{-}\left(z, k_{0}\right)=m_{-}\left(z, k_{0}\right)^{-1} .
$$

As in the continuous case, $\pm M_{ \pm}\left(z, k_{0}\right)$ are Herglotz matrices satisfying

$$
\pm \operatorname{Im}\left(M_{ \pm}\left(z, k_{0}\right)\right)>0, z \in \mathbb{C}_{+}, \quad M_{ \pm}\left(\bar{z}, k_{0}\right)=M_{ \pm}\left(z, k_{0}\right)^{*} .
$$

Next, we introduce the $2 m \times 2 m$ Weyl-Titchmarsh matrix $M\left(z, k_{0}\right)$ associated with the Jacobi operator $H$ in $\ell^{2}(\mathbb{Z})^{m}$ as in (2.47) (with $x_{0} \in \mathbb{R}$ replaced by $k_{0} \in \mathbb{Z}$, etc.) by

$$
M\left(z, k_{0}\right)=\left(M_{j, j^{\prime}}\left(z, k_{0}\right)\right)_{j, j^{\prime}=1,2}, \quad z \in \mathbb{C} \backslash \mathbb{R}, k_{0} \in \mathbb{Z},
$$

where

$$
\begin{aligned}
& M_{1,1}\left(z, k_{0}\right)=\left[M_{-}\left(z, k_{0}\right)-M_{+}\left(z, k_{0}\right)\right]^{-1}, \\
& M_{1,2}\left(z, k_{0}\right)=2^{-1}\left[M_{-}\left(z, k_{0}\right)-M_{+}\left(z, k_{0}\right)\right]^{-1}\left[M_{-}\left(z, k_{0}\right)+M_{+}\left(z, k_{0}\right)\right], \\
& M_{2,1}\left(z, k_{0}\right)=2^{-1}\left[M_{-}\left(z, k_{0}\right)+M_{+}\left(z, k_{0}\right)\right]\left[M_{-}\left(z, k_{0}\right)-M_{+}\left(z, k_{0}\right)\right]^{-1}, \\
& M_{2,2}\left(z, k_{0}\right)=M_{ \pm}\left(z, k_{0}\right)\left[M_{-}\left(z, k_{0}\right)-M_{+}\left(z, k_{0}\right)\right]^{-1} M_{\mp}\left(z, k_{0}\right) .
\end{aligned}
$$

Remark 4.2. We note that the Weyl-Titchmarsh matrix $M\left(z, k_{0}\right)$ is related to the Green's matrix associated with the Jacobi operator $H$ by

$$
M\left(z, k_{0}\right)=\left(\begin{array}{cc}
I_{m} & 0 \\
0 & -A\left(k_{0}\right)
\end{array}\right) \mathcal{M}\left(z, k_{0}\right)\left(\begin{array}{cc}
I_{m} & 0 \\
0 & -A\left(k_{0}\right)
\end{array}\right)+\frac{1}{2}\left(\begin{array}{cc}
0 & I_{m} \\
I_{m} & 0
\end{array}\right),
$$

where

$$
\mathcal{M}\left(z, k_{0}\right)=\left(\begin{array}{cc}
G\left(z, k_{0}, k_{0}\right) & G\left(z, k_{0}, k_{0}+1\right) \\
G\left(z, k_{0}+1, k_{0}\right) & G\left(z, k_{0}+1, k_{0}+1\right)
\end{array}\right) .
$$

With $M\left(z, k_{0}\right)$ defined in (4.32), the analog of Theorem 2.12 holds.

Theorem 4.3. Assume Hypothesis 4.1 and let $k_{0} \in \mathbb{Z}$. Then the $2 m \times 2 m$ WeylTitchmarsh matrix $M\left(z, k_{0}\right)$ for all $z \in \mathbb{C}_{+}$uniquely determines the Jacobi operator $H$ and hence $A=\{A(k)\}_{k \in \mathbb{Z}}$ and $B=\{B(k)\}_{k \in \mathbb{Z}}$.

Perhaps the simplest way to prove Theorem 4.3 is to reduce it to knowledge of $M_{ \pm}\left(z, k_{0}\right)$, and hence by (4.29) and (4.30) to that of $m_{ \pm}\left(z, k_{0}\right)$ for all $z \in \mathbb{C}_{+}$. Using the standard construction of orthogonal matrix-valued polynomials with respect to the normalized measure in the Herglotz representation of $m_{ \pm}\left(z, k_{0}\right)$,

$$
m_{ \pm}\left(z, k_{0}\right)=\int_{\mathbb{R}} d \Omega_{ \pm}\left(\lambda, k_{0}\right)(\lambda-z)^{-1}, \quad z \in \mathbb{C}_{+}, \quad \int_{\mathbb{R}} d \Omega_{ \pm}\left(\lambda, k_{0}\right)=I_{m},
$$


allows one to reconstruct $A(k), B(k), k \in\left[k_{0}, \pm \infty\right) \cap \mathbb{Z}$ from the measures $d \Omega_{ \pm}\left(\lambda, k_{0}\right)$ (cf., e.g., [19, Section VII.2.8]). More precisely,

$$
\begin{aligned}
& A\left(k_{0} \pm k\right)=\int_{\mathbb{R}} \lambda P_{ \pm, k}\left(\lambda, k_{0}\right) d \Omega_{ \pm}\left(\lambda, k_{0}\right) P_{ \pm, k+1}\left(\lambda, k_{0}\right)^{*} \\
& B\left(k_{0} \pm k\right)=\int_{\mathbb{R}} \lambda P_{ \pm, k}\left(\lambda, k_{0}\right) d \Omega_{ \pm}\left(\lambda, k_{0}\right) P_{ \pm, k}\left(\lambda, k_{0}\right)^{*}, \quad k \in \mathbb{N}_{0},
\end{aligned}
$$

where $\left\{P_{ \pm, k}\left(\lambda, k_{0}\right)\right\}_{k \in \mathbb{N}_{0}}$ is an orthonormal system of matrix-valued polynomials with respect to the spectral measure $d \Omega_{ \pm}\left(\lambda, k_{0}\right)$, with $P_{ \pm, k}\left(z, k_{0}\right)$ of degree $k$ in $z$, $P_{ \pm, 0}\left(z, k_{0}\right)=I_{m}$. One verifies,

$$
\begin{aligned}
& P_{+, k}\left(z, k_{0}\right)=\phi\left(z, k_{0}+k, k_{0}-1\right), \\
& P_{-, k}\left(z, k_{0}\right)=\theta\left(z, k_{0}-k, k_{0}\right), \quad k \in\{-1\} \cup \mathbb{N}_{0}, k_{0} \in \mathbb{Z}, z \in \mathbb{C},
\end{aligned}
$$

with $\phi\left(z, k, k_{0}\right)$ and $\theta\left(z, k, k_{0}\right)$ defined in (4.6), (4.7).

In the following we denote by $\lfloor x\rfloor \in \mathbb{Z}$ the largest integer less or equal to $x \in \mathbb{R}$.

The local analog of Theorem 4.3 then reads as follows.

Theorem 4.4. Suppose $0<\varepsilon<\pi$. Let $H_{j}, j=1,2$ be two Jacobi operators in $\ell^{2}(\mathbb{Z})^{m}$, with coefficients $\left\{A_{j, k}\right\}_{k \in \mathbb{Z}},\left\{B_{j, k}\right\}_{k \in \mathbb{Z}}$ satisfying Hypothesis 4.1 , and denote by $M_{j, \pm}\left(z, k_{0}\right)$ the $m \times m$ Weyl-Titchmarsh matrices associated with $H_{j}$, $j=1,2$. If there exists an $N \in \mathbb{N}, N \geq 2$, such that

$$
\left\|M_{1,-}\left(z, k_{0}\right)-M_{2,-}\left(z, k_{0}\right)\right\|_{\mathbb{C}^{m \times m}} \underset{|z| \rightarrow \infty}{=} O\left(|z|^{-N}\right)
$$

along a ray with $\arg (z)=\pi-\varepsilon$, then

$$
\begin{array}{ll}
A_{1}\left(k_{0}-k\right)=A_{2}\left(k_{0}-k\right), & k=0, \ldots,\lfloor N / 2\rfloor-1, \\
B_{1}\left(k_{0}-k\right)=B_{2}\left(k_{0}-k\right), & k=0, \ldots,\lfloor(N-1) / 2\rfloor .
\end{array}
$$

If there exists an $N \in \mathbb{N}, N \geq 2$, such that

$$
\left\|M_{1,+}\left(z, k_{0}\right)-M_{2,+}\left(z, k_{0}\right)\right\|_{\mathbb{C}^{m \times m}} \underset{|z| \rightarrow \infty}{=} O\left(|z|^{-N}\right)
$$

along a ray with $\arg (z)=\pi-\varepsilon$ and

$$
B_{1}\left(k_{0}\right)=B_{2}\left(k_{0}\right)
$$

then

$$
\begin{array}{ll}
A_{1}\left(k_{0}+k\right)=A_{2}\left(k_{0}+k\right), & k=0, \ldots,\lfloor N / 2\rfloor-1, \\
B_{1}\left(k_{0}+k\right)=B_{2}\left(k_{0}+k\right), & k=0, \ldots,\lfloor(N-1) / 2\rfloor .
\end{array}
$$

Proof. Using (4.29), 4.30), and the asymptotic representations

$$
m_{j, \pm}\left(z, k_{0}\right) \underset{|z| \rightarrow \infty}{=}-z^{-1}+O\left(|z|^{-2}\right), \quad j=1,2,
$$

one infers

$$
m_{1, \pm}\left(z, k_{0}\right)-m_{2, \pm}\left(z, k_{0}\right) \underset{|z| \rightarrow \infty}{=} O\left(|z|^{-2}\left(M_{1, \pm}\left(z, k_{0}\right)-M_{2, \pm}\left(z, k_{0}\right)\right)\right) .
$$

Here assumption (4.46) is explicitly needed in the case of the + -sign in $(4.49)$. By hypothesis (4.43), (4.49) implies

$$
m_{1, \pm}\left(z, k_{0}\right)-m_{2, \pm}\left(z, k_{0}\right) \underset{|z| \rightarrow \infty}{=} O\left(|z|^{-(N+2)}\right),
$$


and hence

$$
\int_{\mathbb{R}} d \Omega_{1, \pm}\left(\lambda, k_{0}\right) \lambda^{n}=\int_{\mathbb{R}} d \Omega_{2, \pm}\left(\lambda, k_{0}\right) \lambda^{n}, \quad n=0,1, \ldots, N .
$$

Introducing the orthogonal polynomials $\left\{P_{j, \pm ; k}\left(\lambda, k_{0}\right)\right\}_{k \in \mathbb{N}_{0}}$ associated with the normalized spectral measures $d \Omega_{j, \pm}\left(\lambda, k_{0}\right), j=1,2$, (4.51) implies that

$$
\begin{aligned}
& \int_{\mathbb{R}} \lambda P_{1, \pm ; k}\left(\lambda, k_{0}\right) d \Omega_{1, \pm}\left(\lambda, k_{0}\right) P_{1, \pm ; k+1}\left(\lambda, k_{0}\right)^{*} \\
& =\int_{\mathbb{R}} \lambda P_{2, \pm ; k}\left(\lambda, k_{0}\right) d \Omega_{2, \pm}\left(\lambda, k_{0}\right) P_{2, \pm ; k+1}\left(\lambda, k_{0}\right)^{*}, \quad k=0, \ldots,\lfloor N / 2\rfloor-1,
\end{aligned}
$$

and

$$
\begin{aligned}
& \int_{\mathbb{R}} \lambda P_{1, \pm ; k}\left(\lambda, k_{0}\right) d \Omega_{1, \pm}\left(\lambda, k_{0}\right) P_{1, \pm ; k}\left(\lambda, k_{0}\right)^{*} \\
& =\int_{\mathbb{R}} \lambda P_{2, \pm ; k}\left(\lambda, k_{0}\right) d \Omega_{2, \pm}\left(\lambda, k_{0}\right) P_{2, \pm ; k}\left(\lambda, k_{0}\right)^{*}, \quad k=0, \ldots,\lfloor(N-1) / 2\rfloor,
\end{aligned}
$$

which proves (4.44) using (4.40).

Of course, one can permit $\varepsilon=0$ and $\varepsilon=\pi$ in Theorem 4.4 (and in Theorem 4.8 below) as long as $|z|>\max _{j=1,2}\left\|H_{j}\right\|$.

Remark 4.5. The apparent asymmetry with respect to the additional condition (4.46) in connection with $M_{j,+}\left(z, k_{0}\right)$ is due to the fact that for fixed $k_{0} \in \mathbb{Z}$, the map

$$
\left(m_{+}\left(z, k_{0}\right), B\left(k_{0}\right)\right) \mapsto M_{+}\left(z, k_{0}\right), \quad z \in \mathbb{C}
$$

given by (4.29) is not injective and hence knowledge of $M_{+}\left(z, k_{0}\right)$ does not determine $m_{+}\left(z, k_{0}\right)$ uniquely. This follows from the explicit cancellation of $B\left(k_{0}\right)$ in $(4.29)$ as $|z| \rightarrow \infty$ in sharp contrast to the asymptotic behavior of $M_{-}\left(z, k_{0}\right)$ in (4.30), as described explicitly in 4.59 ) and (4.60) below.

Given these preliminaries and introducing the diagonal Green's matrix by

$$
g(z, k)=G(z, k, k), \quad z \in \mathbb{C} \backslash \mathbb{R}, k \in \mathbb{Z},
$$

we can now formulate the analog of the uniqueness result, Theorem 3.7, for Jacobi operators.

Theorem 4.6. Assume Hypothesis 4.1 and let $k_{0} \in \mathbb{Z}$. Then any of the following three sets of data

(i) $g\left(z, k_{0}\right)$ and $G\left(z, k_{0}, k_{0}+1\right)$ for all $z \in \mathbb{C}_{+}$;

(ii) $g\left(z, k_{0}\right)$ and $\left[G\left(z, k_{0}, k_{0}+1\right)+G\left(z, k_{0}+1, k_{0}\right)\right]$ for all $z \in \mathbb{C}_{+}$;

(iii) $g\left(z, k_{0}\right), g\left(z, k_{0}+1\right)$ for all $z \in \mathbb{C}_{+}$and $A\left(k_{0}\right)$;

uniquely determines the matrix-valued Jacobi operator $H$ and hence $A=\{A(k)\}_{k \in \mathbb{Z}}$ and $B=\{B(k)\}_{k \in \mathbb{Z}}$.

Proof. Case (i). A direct computation utilizing formulas (4.28), (4.55) shows that

$$
\begin{aligned}
g\left(z, k_{0}\right) & =\left[M_{-}\left(z, k_{0}\right)-M_{+}\left(z, k_{0}\right)\right]^{-1} \\
G\left(z, k_{0}, k_{0}+1\right) & =-\left[M_{-}\left(z, k_{0}\right)-M_{+}\left(z, k_{0}\right)\right]^{-1} M_{+}\left(z, k_{0}\right) A\left(k_{0}\right)^{-1} .
\end{aligned}
$$


In addition, the matrix $A\left(k_{0}\right)$ can be read off from the asymptotic expansion of $G\left(z, k_{0}, k_{0}+1, z\right)$ as $z \rightarrow \infty$ by a simple Neumann series-type argument. Indeed, by $(4.20)$

$$
m_{ \pm}\left(z, k_{0}\right)=-z^{-1} I_{m}-z^{-2} B\left(k_{0}\right)-z^{-3}\left(A\left(k_{0}\right)^{2}+B\left(k_{0}\right)^{2}\right)+O\left(|z|^{-4}\right)
$$

and hence by $(4.29)$ and $(4.30$

$$
\begin{aligned}
& M_{+}\left(z, k_{0}\right) \underset{|z| \rightarrow \infty}{=}-z^{-1} A\left(k_{0}\right)^{2}+O\left(|z|^{-2}\right), \\
& M_{-}\left(z, k_{0}\right) \underset{|z| \rightarrow \infty}{=}-z I_{m}+B\left(k_{0}\right)+z^{-1} A\left(k_{0}\right)^{2}+O\left(|z|^{-2}\right)
\end{aligned}
$$

along any ray with $\arg (z)=\pi-\varepsilon, 0<\varepsilon<\pi$. Therefore, combining 4.56), (4.57), (4.59), and (4.60) one infers that

$$
G\left(z, k_{0}, k_{0}+1\right) \underset{|z| \rightarrow \infty}{=}-z^{-2} A\left(k_{0}\right)+O\left(|z|^{-3}\right), \quad k_{0} \in \mathbb{Z}
$$

along any ray with $\arg (z)=\pi-\varepsilon, 0<\varepsilon<\pi$, in complete analogy with the corresponding scalar case (see, e.g., [135, [136, Sect. 6.1]). Given $A\left(k_{0}\right)$ and using (4.56) one can easily solve (4.57) for $M_{ \pm}\left(z, k_{0}\right)$,

$$
\begin{aligned}
M_{+}\left(z, k_{0}\right) & =-g\left(z, k_{0}\right)^{-1} G\left(z, k_{0}, k_{0}+1\right) A\left(k_{0}\right), \\
M_{-}\left(z, k_{0}\right) & =M_{+}\left(z, k_{0}\right)+g\left(z, k_{0}\right)^{-1} \\
& =g\left(z, k_{0}\right)^{-1}\left(I_{m}-G\left(z, k_{0}, k_{0}+1\right) A\left(k_{0}\right)\right),
\end{aligned}
$$

which in turn determines $A(k), B(k)$ for all $k \in \mathbb{Z}$ by Theorem 4.3 .

Case (ii). The data (ii) are perhaps the closest discrete analog of the continuous version in Theorem 3.7. As before (cf. (4.61)), one can determine $A\left(k_{0}\right)$ from the asymptotic expansion of $G\left(z, k_{0}, k_{0}+1\right)+G\left(z, k_{0}+1, k_{0}\right)$,

$$
G\left(z, k_{0}, k_{0}+1\right)+G\left(z, k_{0}+1, k_{0}\right) \underset{|z| \rightarrow \infty}{=}-2 z^{-2} A\left(k_{0}\right)+O\left(|z|^{-3}\right), \quad k_{0} \in \mathbb{Z} .
$$

Next, using (4.28), one observes that $M_{+}\left(z, k_{0}\right)$ is the unique solution of the following Sylvester-type matrix equation

$$
\begin{aligned}
& G\left(z, k_{0}+1, k_{0}\right)+G\left(z, k_{0}, k_{0}+1\right) \\
& =-A\left(k_{0}\right)^{-1} M_{+}\left(z, k_{0}\right) g\left(z, k_{0}\right)-g\left(z, k_{0}\right) M_{+}\left(z, k_{0}\right) A\left(k_{0}\right)^{-1}
\end{aligned}
$$

(cf. (3.33)) by Lemmas 3.3 and 3.4. Determining $M_{-}\left(z, k_{0}\right)$ from (4.63) completes the proof of case (ii) by Theorem 4.3 as in case (i).

Case (iii). Since $A\left(k_{0}\right)$ is known, the data (iii), (4.28), and (4.55) imply

$$
g\left(z, k_{0}+1\right)=A\left(k_{0}\right)^{-1} M_{+}\left(z, k_{0}\right)\left[M_{-}\left(z, k_{0}\right)-M_{+}\left(z, k_{0}\right)\right]^{-1} M_{-}\left(z, k_{0}\right) A\left(k_{0}\right)^{-1} .
$$

Together with formula (4.56) for $g\left(z, k_{0}\right)$ this leads to the following equation for $M_{+}\left(z, k_{0}\right)$,

$$
M_{+}\left(z, k_{0}\right)+M_{+}\left(z, k_{0}\right) g\left(z, k_{0}\right) M_{+}\left(z, k_{0}\right)=A\left(k_{0}\right) g\left(z, k_{0}+1\right) A\left(k_{0}\right) .
$$

Using the asymptotics (4.59), (4.60) and the representation

$$
g(z, k)=-z^{-1} I_{m}+O\left(|z|^{-2}\right), \quad k \in \mathbb{Z},
$$

one infers

$$
\left\|M_{+}\left(z, k_{0}\right)\right\|_{\mathbb{C}^{m \times m}}<1, \quad\left\|A\left(k_{0}\right) g\left(z, k_{0}+1\right) A\left(k_{0}\right)\right\|_{\mathbb{C}^{m \times m}}<\frac{1}{2},
$$




$$
\text { and }\left\|g\left(z, k_{0}\right)\right\|_{\mathbb{C}^{m \times m}}<\frac{1}{2}
$$

for $\operatorname{Im}(z)>0$ sufficiently large. Thus, by Lemma 3.5, $M_{+}\left(z, k_{0}\right)$ is uniquely determined by the data (iii) for $\operatorname{Im}(z)>0$ large enough and hence for all $z \in \mathbb{C}_{+}$ by analytic continuation. The Weyl matrix $M_{-}\left(z, k_{0}\right)$ is then determined by (cf. (4.63))

$$
M_{-}\left(z, k_{0}\right)=M_{+}\left(z, k_{0}\right)+g\left(z, k_{0}\right)^{-1},
$$

completing the proof.

Remark 4.7. We note that $M_{+}\left(z, k_{0}\right)$ can explicitly be represented by the contour integral (cf. (3.34))

$$
\begin{aligned}
& M_{+}\left(z, k_{0}\right) \\
& =(2 \pi i)^{-1} \oint_{\Gamma} d \zeta\left(g\left(z, k_{0}\right)-\zeta A\left(k_{0}\right)^{-1}\right)^{-1}\left[G\left(z, k_{0}, k_{0}+1\right)+G\left(z, k_{0}+1, k_{0}\right)\right] \times \\
& \times\left(g\left(z, k_{0}\right)+\zeta A\left(k_{0}\right)^{-1}\right)^{-1} .
\end{aligned}
$$

Here $\Gamma$ is a closed clockwise oriented Jordan contour such that $\operatorname{spec}\left(A\left(k_{0}\right) g\left(z, k_{0}\right)\right)$ has winding number +1 and $\operatorname{spec}\left(-g\left(z, k_{0}\right) A\left(k_{0}\right)\right)$ has winding number 0 with respect to $\Gamma$. Without loss of generality we may assume that $\Gamma \subset \mathbb{C}_{+}$. The existence of such a contour $\Gamma$ follows from the observation that

$$
\operatorname{spec}\left(g\left(z, k_{0}\right) A\left(k_{0}\right)\right)=\operatorname{spec}\left(A\left(k_{0}\right)^{1 / 2} g\left(z, k_{0}\right) A\left(k_{0}\right)^{1 / 2}\right) \subset \mathbb{C}_{+}, \quad z \in \mathbb{C}_{+}
$$

by Lemma 3.4 .

The special scalar case $m=1$ of Theorem 4.6 is known and has recently been derived in 135] (see also [136, Sect. 2.7]. Condition (iii) in Theorem 4.6 is specific to the Jacobi case. In the corresponding Schrödinger case, the corresponding set of data does not even uniquely determine the potential in the scalar case $m=1$ (see, e.g., [57, 135]).

The local analog of Theorem 3.8 in the discrete context then reads as follows.

Theorem 4.8. Suppose $0<\varepsilon<\pi$. Let $H_{j}, j=1,2$ be two Jacobi operators in $\ell^{2}(\mathbb{Z})^{m}$, with coefficients $A_{j}=\left\{A_{j, k}\right\}_{k \in \mathbb{Z}}, B_{j}=\left\{B_{j, k}\right\}_{k \in \mathbb{Z}}$ satisfying Hypothesis 4.1. and denote by $G_{j}(z, k, \ell), g_{j}(z, k)=G_{j}(z, k, k)(k, \ell \in \mathbb{Z})$ the Green's matrices associated with $H_{j}, j=1,2$. Moreover, assume there exists an $N \in \mathbb{N}$, $N \geq 2$, such that one of the following conditions holds.

(i)

$$
\begin{aligned}
& \left\|g_{1}\left(z, k_{0}\right)-g_{2}\left(z, k_{0}\right)\right\|_{\mathbb{C}^{m \times m}}+\left\|G_{1}\left(z, k_{0}, k_{0}+1\right)-G_{2}\left(z, k_{0}, k_{0}+1\right)\right\|_{\mathbb{C}^{m \times m}} \\
& \underset{|z| \rightarrow \infty}{=} O\left(|z|^{-(N+2)}\right) ;
\end{aligned}
$$

(ii)

$$
\begin{aligned}
& \left\|g_{1}\left(z, k_{0}\right)-g_{2}\left(z, k_{0}\right)\right\|_{\mathbb{C}^{m \times m}}+\| G_{1}\left(z, k_{0}, k_{0}+1\right)-G_{2}\left(z, k_{0}, k_{0}+1\right) \\
& +G_{1}\left(z, k_{0}+1, k_{0}\right)-G_{2}\left(z, k_{0}+1, k_{0}\right) \|_{\mathbb{C}^{m \times m}} \underset{|z| \rightarrow \infty}{=} O\left(|z|^{-(N+2)}\right) ;
\end{aligned}
$$

(iii) $A_{1}\left(k_{0}\right)=A_{2}\left(k_{0}\right)$ is given and

$\left\|g_{1}\left(z, k_{0}\right)-g_{2}\left(z, k_{0}\right)\right\|_{\mathbb{C}^{m \times m}}+\left\|g_{1}\left(z, k_{0}+1\right)-g_{2}\left(z, k_{0}+1\right)\right\|_{\mathbb{C}^{m \times m}} \underset{|z| \rightarrow \infty}{=} O\left(|z|^{-(N)}\right)$ 
along a ray with $\arg (z)=\pi-\varepsilon$. Then

$$
\begin{aligned}
& A_{1}\left(k_{0} \pm k\right)=A_{2}\left(k_{0} \pm k\right), \quad k=0, \ldots,\lfloor N / 2\rfloor-1, \\
& B_{1}\left(k_{0} \pm k\right)=B_{2}\left(k_{0} \pm k\right), \quad k=0, \ldots,\lfloor(N-1) / 2\rfloor .
\end{aligned}
$$

Proof. Case (i). Since $N \geq 2$ in (4.73), (4.61) implies that $A_{1}\left(k_{0}\right)=A_{2}\left(k_{0}\right)$ and we denote the latter by $A\left(k_{0}\right)$. By (4.62) the $m \times m$ Weyl-Titchmarsh matrices $M_{j,+}\left(z, k_{0}\right)$ associated with $H_{j}, j=1,2$ can be represented as

$$
M_{j,+}\left(z, k_{0}\right)=-g_{j}\left(z, k_{0}\right)^{-1} G_{j}\left(z, k_{0}, k_{0}+1\right) A\left(k_{0}\right), \quad j=1,2
$$

and they admit the estimate

$$
\begin{aligned}
\| & M_{1,+}\left(z, k_{0}\right)-M_{2,+}\left(z, k_{0}\right) \|_{\mathbb{C}^{m \times m}} \\
\leq & \left\|A\left(k_{0}\right)\right\|_{\mathbb{C}^{m \times m}}\left\|g_{1}\left(z, k_{0}\right)^{-1} G_{1}\left(z, k_{0}, k_{0}+1\right)-g_{2}\left(z, k_{0}\right)^{-1} G_{2}\left(z, k_{0}, k_{0}+1\right)\right\|_{\mathbb{C}^{m \times m}} \\
\leq & \left\|A\left(k_{0}\right)\right\|_{\mathbb{C}^{m \times m}}\left\|g_{1}\left(z, k_{0}\right)^{-1}\right\|_{\mathbb{C}^{m \times m}}\left\|G_{1}\left(z, k_{0}, k_{0}+1\right)-G_{2}\left(z, k_{0}, k_{0}+1\right)\right\|_{\mathbb{C}^{m \times m}} \\
& +\left\|A\left(k_{0}\right)\right\|_{\mathbb{C}^{m \times m}}\left\|g_{1}\left(z, k_{0}\right)^{-1}-g_{2}\left(z, k_{0}\right)^{-1}\right\|_{\mathbb{C}^{m \times m}}\left\|G_{2}\left(z, k_{0}, k_{0}+1\right)\right\|_{\mathbb{C}^{m \times m}} \\
\leq & \left\|A\left(k_{0}\right)\right\|_{\mathbb{C}^{m \times m}}\left\|g_{1}\left(z, k_{0}\right)^{-1}\right\|_{\mathbb{C}^{m \times m}}\left\|G_{1}\left(z, k_{0}, k_{0}+1\right)-G_{2}\left(z, k_{0}, k_{0}+1\right)\right\|_{\mathbb{C}^{m \times m}} \\
& +\left\|A\left(k_{0}\right)\right\|_{\mathbb{C}^{m \times m}}\left\|g_{1}\left(z, k_{0}\right)^{-1}\right\|_{\mathbb{C}^{m \times m}}\left\|g_{1}\left(z, k_{0}\right)-g_{2}\left(z, k_{0}\right)\right\|_{\mathbb{C}^{m \times m}} \times \\
& \times\left\|g_{2}\left(z, k_{0}\right)^{-1}\right\|_{\mathbb{C}^{m \times m}}\left\|G_{2}\left(z, k_{0}, k_{0}+1\right)\right\|_{\mathbb{C}^{m \times m}} .
\end{aligned}
$$

Using the asymptotics

$$
\begin{gathered}
g_{j}\left(z, k_{0}\right) \underset{|z| \rightarrow \infty}{=}-z^{-1} I_{m}+O\left(|z|^{-2}\right), \quad j=1,2, \\
G_{j}\left(z, k_{0}, k_{0}+1\right) \underset{|z| \rightarrow \infty}{=}-z^{-2} A\left(k_{0}\right)+O\left(|z|^{-3}\right), \quad j=1,2,
\end{gathered}
$$

the estimate (4.78) combined with (4.73) proves

$$
\left\|M_{1,+}\left(z, k_{0}\right)-M_{2,+}\left(z, k_{0}\right)\right\|_{\mathbb{C}^{m \times m}} \underset{|z| \rightarrow \infty}{=} O\left(|z|^{-(N+1)}\right) .
$$

Since

$$
M_{j,-}\left(z, k_{0}\right)=M_{j,+}\left(z, k_{0}\right)+g_{j}\left(z, k_{0}\right)^{-1}, \quad j=1,2,
$$

(4.73) and (4.81) yield

$$
\left\|M_{1,-}\left(z, k_{0}\right)-M_{2,-}\left(z, k_{0}\right)\right\|_{\mathbb{C}^{m \times m}} \underset{|z| \rightarrow \infty}{=} O\left(|z|^{-N}\right) .
$$

(4.44) and (4.83) yield

$$
B_{1}\left(k_{0}\right)=B_{2}\left(k_{0}\right) .
$$

The asymptotic expansions (4.81) and (4.83) together with (4.84) then prove (4.76) by Theorem 4.4 .

Case (ii). Since $N \geq 2$ in (4.74), (4.61) again implies that $A_{1}\left(k_{0}\right)=A_{2}\left(k_{0}\right)$ and we denote the latter by $A\left(k_{0}\right)$. Given $\varepsilon \in(0, \pi)$, let $\gamma$ be a closed clockwise oriented Jordan contour

$$
\gamma \subset\{z \in \mathbb{C} \mid \operatorname{Re}(z)>0\}
$$

such that $\operatorname{spec}\left(A\left(k_{0}\right)\right)$ has winding number +1 and

$$
e^{i \varepsilon} \gamma \subset \mathbb{C}_{+} \text {. }
$$


Next, given $z \in \mathbb{C}_{+}, \arg (z)=\pi-\varepsilon$, introduce the contour $\Gamma(z)$ in the upper half-plane by

$$
\Gamma(z)=-z^{-1} \gamma
$$

Using (4.79) one concludes that $\operatorname{spec}\left(A\left(k_{0}\right)^{1 / 2} g_{j}\left(z, k_{0}\right) A\left(k_{0}\right)^{1 / 2}\right)$ has winding number +1 with respect to the contour $\Gamma(z)$ if $z$ lies on the ray $\arg (z)=\pi-\varepsilon$ for $|z|$ sufficiently large. For such $z$ the matrix-valued functions $M_{j,+}\left(z, k_{0}\right)$ admit the representation (cf. (4.71))

$$
\begin{aligned}
M_{j,+}\left(z, k_{0}\right)=(2 \pi i)^{-1} \oint_{\Gamma(z)} & d \zeta \widehat{G}_{j}\left(z, k_{0}, \zeta\right)\left[G_{j}\left(z, k_{0}, k_{0}+1\right)+G_{j}\left(z, k_{0}+1, k_{0}\right)\right] \times \\
& \times \widehat{G}_{j}\left(z, k_{0},-\zeta\right), \quad j=1,2
\end{aligned}
$$

where

$$
\begin{array}{r}
\widehat{G}_{j}\left(z, k_{0}, \zeta\right)=A\left(k_{0}\right)^{1 / 2}\left(A\left(k_{0}\right)^{1 / 2} g_{j}\left(z, k_{0}\right) A\left(k_{0}\right)^{1 / 2}-\zeta\right)^{-1} A\left(k_{0}\right)^{1 / 2}, \\
j=1,2, \zeta \in \Gamma(z) .
\end{array}
$$

Introducing

$$
f_{j}\left(z, k_{0}\right)=g_{j}\left(z, k_{0}\right)+z^{-1} I_{m}, \quad j=1,2,
$$

one infers by (4.79) that

$$
f_{j}\left(z, k_{0}\right) \underset{|z| \rightarrow \infty}{=} O\left(|z|^{-2}\right), \quad j=1,2 .
$$

The asymptotics

$$
\begin{aligned}
& \sup _{\zeta \in \Gamma(z)}\left\|\left(-z^{-1} A\left(k_{0}\right)+A\left(k_{0}\right)^{1 / 2} f_{j}\left(z, k_{0}\right) A\left(k_{0}\right)^{1 / 2} \mp \zeta\right)^{-1}\right\|_{\mathbb{C}^{m \times m}} \\
& =|z| \sup _{\zeta \in \Gamma(z)}\left\|\left(A\left(k_{0}\right)-z A\left(k_{0}\right)^{1 / 2} f_{j}\left(z, k_{0}\right) A\left(k_{0}\right)^{1 / 2} \pm z \zeta\right)^{-1}\right\|_{\mathbb{C}^{m \times m}} \\
& =|z| \sup _{\zeta \in \gamma}\left\|\left(A\left(k_{0}\right) \mp \zeta\right)^{-1}\right\|_{\mathbb{C}^{m \times m}} \times \\
& \sup _{\zeta \in \gamma}\left\|\left(I_{m}-z\left(A\left(k_{0}\right) \mp \zeta\right)^{-1} A\left(k_{0}\right)^{1 / 2} f_{j}\left(z, k_{0}\right) A\left(k_{0}\right)^{1 / 2}\right)^{-1}\right\|_{\mathbb{C}^{m \times m}} \\
& \underset{|z| \rightarrow \infty}{=} O(|z|)
\end{aligned}
$$

along the ray $\arg (z)=\pi-\varepsilon$, and the representation

$$
\begin{array}{r}
\widehat{G}_{j}\left(z, k_{0}, \zeta\right)=A\left(k_{0}\right)^{1 / 2}\left(-z^{-1} A\left(k_{0}\right)+A\left(k_{0}\right)^{1 / 2} f_{j}\left(z, k_{0}\right) A\left(k_{0}\right)^{1 / 2}-\zeta\right)^{-1} A\left(k_{0}\right)^{1 / 2} \\
j=1,2
\end{array}
$$

then yield

$$
\left\|\widehat{G}_{j}\left(z, k_{0}, \pm \zeta\right)\right\|_{\mathbb{C}^{m \times m}} \underset{|z| \rightarrow \infty}{=} O(|z|), \quad \zeta \in \Gamma(z), \quad j=1,2,
$$

along the ray $\arg (z)=\pi-\varepsilon$, uniformly with respect to $\zeta \in \Gamma(z)$. As a consequence of (4.94) and hypothesis (4.74) we have

$$
\left\|\widehat{G}_{1}\left(z, k_{0}, \pm \zeta\right)-\widehat{G}_{2}\left(z, k_{0}, \pm \zeta\right)\right\|_{\mathbb{C}^{m \times m}} \underset{|z| \rightarrow \infty}{=} O\left(|z|^{-N}\right), \quad \zeta \in \Gamma(z)
$$

along the ray $\arg (z)=\pi-\varepsilon$, uniformly in $\zeta \in \Gamma(z)$. Using (4.88), (4.94), and (4.95) one concludes that

$$
\left\|M_{1,+}\left(z, k_{0}\right)-M_{2,+}\left(z, k_{0}\right)\right\|_{\mathbb{C}^{m \times m}} \underset{|z| \rightarrow \infty}{=} O\left(|z|^{-(N+1)}\right)
$$


and hence by $(4.76)$ and $(4.74)$ that

$$
\left\|M_{1,-}\left(z, k_{0}\right)-M_{2,-}\left(z, k_{0}\right)\right\|_{\mathbb{C}^{m \times m}} \underset{|z| \rightarrow \infty}{=} O\left(|z|^{-N}\right)
$$

along the $\operatorname{ray} \arg (z)=\pi-\varepsilon$. This proves (4.76) by Theorem 4.4 .

Case (iii). One observes that $M_{j,+}\left(z, k_{0}\right)$ satisfy (c.f. (4.67))

$M_{j,+}\left(z, k_{0}\right)+M_{j,+}\left(z, k_{0}\right) g_{j}\left(z, k_{0}\right) M_{j,+}\left(z, k_{0}\right)=A\left(k_{0}\right) g_{j}\left(z, k_{0}+1\right) A\left(k_{0}\right), \quad j=1,2$.

Taking into account the asymptotic expansions $(4.79)$ and $(4.80)$, hypothesis 4.75 implies (4.96) and (4.97) by Corollary 3.6. This proves (4.76) applying Theorem 4.4 again.

Without going into further details, we note that the methods employed in this section can easily be adapted to the case of uniformly bounded operator coefficients for Jacobi operators (i.e., for some $C>0$ and all $k \in \mathbb{Z},\|A(k)\|_{\mathcal{B}(\mathcal{H})}+\|B(k)\|_{\mathcal{B}(\mathcal{H})} \leq$ $C, A(k)^{-1} \in \mathcal{B}(\mathcal{H}), 0<A(k), B(k)=B(k)^{*}$, for some complex separable Hilbert space $\mathcal{H})$.

Acknowledgements. We are indebted to Steve Clark, Don Hinton, Boris Levitan, Mark Malamud, Fedor Rofe-Beketov, Alexander Sakhnovich, Lev Sakhnovich, and Barry Simon for discussions and valuable hints regarding the literature. F.G. and A.K. thank C. Peck and T. Tombrello for the hospitality of Caltech during the final stages of this work.

\section{REFERENCES}

[1] Z. S. Agranovich and V.A. Marchenko, The Inverse Problem of Scattering Theory, Gordon and Breach, New York, 1963.

[2] B. P. Allakhverdiev, Extensions of symmetric Schrödinger operators with matrix coefficients, Izvest. Math. 59, 45-62 (1995).

[3] D. Alpay and I. Gohberg, Inverse spectral problems for difference operators with rational scattering matrix function, Integr. Equat. Oper. Th. 20, 125-170 (1994).

[4] D. Alpay and I. Gohberg, Inverse spectral problem for differential operators with rational scattering matrix functions, J. Diff. Eq. 118, 1-19 (1995).

[5] D. Alpay and I. Gohberg, Inverse problem for Sturm-Liouville operators with rational reflection coefficient, Integr. Equat. Oper. Th. 30, 317-325 (1998).

[6] A. I. Aptekarev and E. M. Nikishin, The scattering problem for a discrete Sturm-Liouville problem, Math. USSR Sborn. 49, 325-355 (1984).

[7] D. Z. Arov and H. Dym, J-inner matrix functions, interpolation and inverse problems for canonical systems, I: foundations, Integr. equ. oper. theory 29, 373-454 (1997).

[8] D. Z. Arov and H. Dym, J-inner matrix functions, interpolation and inverse problems for canonical systems,II: the inverse monodromy problem, Integr. equ. oper. theory 36, 11-70 (2000).

[9] D. Z. Arov and H. Dym, J-inner matrix functions, interpolation and inverse problems for canonical systems,III: more on the inverse monodromy problem, Integr. equ. oper. theory 36, 127-181 (2000).

[10] N. Asano and Y. Kato, The Marchenko equation of the Hilbert-Schmidt type for the $N \times N$ Zakharov-Shabat operator, Progress Theoret. Phys. 80, 774-792 (1988).

[11] N. Asano and Y. Kato, Algebraic and Spectral Methods for Nonlinear Wave Equations, Longman, New York, 1990.

[12] F. V. Atkinson, Discrete and Continuous Boundary Problems, Academic Press, New York, 1964.

[13] F. V. Atkinson, On the order of magnitude of Titchmarsh-Weyl functions, Diff. Integral Eqs. 1, 79-96 (1988). 
[14] F. V. Atkinson, Asymptotics of the Titchmarsh-Weyl function in the matrix case, unpublished manuscript.

[15] R. Benguria and M. Loss, A simple proof of a theorem of Laptev and Weidl, Math. Res. Lett. 7, 195-203 (2000).

[16] C. Bennewitz, A proof of the local Borg-Marchenko theorem, Comm. Math. Phys., to appear.

[17] Ju. M. Berezanskii, On the uniqueness of the determination of Schrödinger's equation from its spectral function, Sov. Math. Dokl. 93, 591-594 (1953). (Russian).

[18] Ju. M. Berezanskii, The uniqueness theorem in the inverse problem of spectral analysis for the Schrödinger operator, Amer. Math. Soc. Transl., Ser. 2, 35, 167-235 (1964).

[19] Ju. Berezanskii, Expansions in Eigenfunctions of Selfadjoint Operators, Transl. Math. Mongraphs, Vol. 17, Amer. Math. Soc., Providence, R.I., 1968.

[20] Yu. M. Berezanskii, M. I. Gekhtman, and M. E. Shmoish, Integration of some chains of nonlinear difference equations by the method of the inverse spectral problem, Ukrain. Math. J. 38, 74-78 (1986).

[21] Yu. M. Berezanskii and M. I. Gekhtman, Inverse problem of the spectral analysis and nonabelian chains of nonlinear equations, Ukrain. Math. J. 42, 645-658 (1990).

[22] R. Bhatia, Matrix Analysis, Springer, New York, 1997.

[23] R. Bhatia, C. Davies, and A. McIntosh, Perturbation of spectral subspaces and solution of linear operator equations, Linear Algebra Appls. 52/53, 45-67 (1983).

[24] G. Borg, Uniqueness theorems in the spectral theory of $y^{\prime \prime}+(\lambda-q(x)) y=0$, Proc. 11th Scandinavian Congress of Mathematicians, Johan Grundt Tanums Forlag, Oslo, 1952, pp. 276-287.

[25] R. Carlson, Large eigenvalues and trace formulas for matrix Sturm-Liouville problems, SIAM J. Math. Anal. 30, 949-962 (1999).

[26] R. Carlson, Compactness of Floquet isospectral sets for the matrix Hill's equation, Proc. Amer. Math. Soc. 128, 2933-2941 (2000).

[27] R. Carlson, Eigenvalue estimates and trace formulas for the matrix Hill's equation, J. Diff. Eq. 167, 211-244 (2000).

[28] R. Carlson, An inverse problem for the matrix Schrödinger equation, preprint, 2000.

[29] R. Carmona and J. Lacroix, Spectral Theory of Random Schrödinger operators, Birkhäuser, Boston, MA, 1990.

[30] I. Cherednik, Basic Methods of Soliton Theory, World Scientific, Singapore, 1996.

[31] H.-H. Chern, On the construction of isospectral vectorial Sturm-Liouville differential equations, preprint, 1998.

[32] H.-H. Chern and C-L. Shen, On the n-dimensional Ambarzumyan's theorem, Inverse Problems 13, 15-18 (1997).

[33] S. L. Clark, A criterion for absolute continuity of the continuous spectrum of a Hamiltonian system, J. Math. Anal. Appl. 151, 108-128 (1990).

[34] S. L. Clark, Asymptotic behavior of the Titchmarsh-Weyl coefficient for a coupled second order system, in "Ordinary and Delay Differential Equations", J. Wiener and J. K. Hale (eds.), Longman, New York, 1992, pp. 24-28.

[35] S. L. Clark, On the absolutely continuous spectrum of a vector-matrix Dirac system, Proc. Roy. Soc. Edinburgh 124A, 253-262 (1994).

[36] S. Clark and F. Gesztesy, Weyl-Titchmarsh $M$-function asymptotics for matrix-valued Schrödinger operators, Proc. London Math. Soc., to appear.

[37] S. Clark and F. Gesztesy, Weyl-Titchmarsh $M$-function asymptotics, local uniqueness results, trace formulas, and Borg-type theorems for Dirac operators, preprint, 2001.

[38] S. Clark, F. Gesztesy, H. Holden, and B. M. Levitan, Borg-type theorems for matrix-valued Schrödinger and Dirac operators, J. Diff. Eqs. 167, 181-210 (2000).

[39] S. Clark and D. Hinton, A Liapunov inequality for linear Hamiltonian systems, Math. Inequ. Appl. 1, 201-209 (1998).

[40] P. Delsarte, Y. V. Genin, and Y. G. Kamp, Orthogonal polynomial matrices on the unit circle, IEEE Trans. Circ. Syst. 25, 149-160 (1978).

[41] V. I. Derguzov, The spectrum of Hamilton's operator with periodic coefficients, Vestnik Leningrad Univ. Math. 12, 280-285 (1980).

[42] B. Després, The Borg theorem for the vectorial Hill's equation, Inverse Probl. 11, 97-121 (1995).

[43] L. A. Dickey, Soliton Equations and Hamiltonian Systems, World Scientific, Singapore, 1991. 
[44] B. A. Dubrovin, Completely integrable Hamiltonian Systems associated with Matrix operators and Abelian varieties, Funct. Anal. Appl. 11, 265-277 (1977).

[45] B. A. Dubrovin, Matrix finite-zone operators, Revs. Sci. Tech. 23, 20-50 (1983).

[46] A. J. Duran and W. Van Assche, Orthogonal matrix polynomials and higher-order recurrence relations, Lin. Algebra Appl. 219, 261-280 (1995).

[47] A. J. Duran and P. Lopez-Rodriguez, Orthogonal matrix polynomials: zeroe and Blumenthal's theorem, J. Approx. Th. 84, 96-118 (1996).

[48] M. Fukushima, A spectral representation on ordinary linear difference equation with operatorvalued coefficients of the second order, J. Math. Phys. 17, 1084-1072 (1976).

[49] M. G. Gasymov, The inverse scattering problem for a system of Dirac equations of order $2 n$, Trans. Moscow Math. Soc. 19, 41-119 (1968).

[50] M. G. Gasymov and B. M. Levitan, The inverse problem for a Dirac system, Sov. Math. Dokl. 7, 495-499 (1966).

[51] I. M. Gel'fand and L. A. Dikii, The resolvent and Hamiltonian systems, Funct. Anal. Appl. 11, 93-105 (1977).

[52] I. M. Gel'fand and V. B. Lidskij, On the structure of the regions of stability of linear canonical systems of differential equations with periodic coefficients, in "Izrail M. Gelfand, Collected Papers", Vol. I, S. G. Gindikin, V. W. Guillemin, A. A. Kirillov, B. Kostant, S. Sternberg (eds.), Springer, Berlin, 1987, pp. 466-504. (Amer. Math. Soc. Transl. (2) 8, 143-181 (1958).)

[53] J. S. Geronimo, Matrix orthogonal polynomials on the unit circle, J. Math. Phys. 22, 13591365 (1981).

[54] J. S. Geronimo, Scattering theory and matrix orthogonal polynomials on the real line, Circuits Syst. Signal Process. 1, 471-495 (1982).

[55] F. Gesztesy and H. Holden, On trace formulas for Schrödinger-type operators, in Multiparticle Quantum Scattering with Applications to Nuclear, Atomic and Molecular Physics, D. G. Truhlar and B. Simon (eds.), Springer, New York, 1997, pp. 121-145.

[56] F. Gesztesy, K. A. Makarov, and S. N. Naboko, The spectral shift operator, in Mathematical Results in Quantum Mechanics, J. Dittrich, P. Exner, and M. Tater (eds.), Operator Theory: Advances and Applications, Vol. 108, Birkhäuser, Basel, 1999, pp. 59-90.

[57] F. Gesztesy and B. Simon, Uniqueness theorems in inverse spectral theory for one-dimensinal Schrödinger operators, Trans. Amer. Math. Soc. 348, 349-373 (1996).

[58] F. Gesztesy and B. Simon, The $\xi$ function, Acta Math. 176, 49-71 (1996).

[59] F. Gesztesy and B. Simon, A new approach to inverse spectral theory, II. General real potentials and the connection to the spectral measure, Ann. of Math. 152, 593-643 (2000).

[60] F. Gesztesy and B. Simon, On local Borg-Marchenko uniqueness results, Commun. Math. Phys. 211, 273-287 (2000).

[61] F. Gesztesy and E. Tsekanovskii, On matrix-valued Herglotz functions, Math. Nachr. 218, 61-138 (2000).

[62] I. Gohberg, M. A. Kaashoek, and A. L. Sakhnovich, Sturm-Liouville systems with rational Weyl functions: explicit formulas and applications, Integral Eq. Operator Th. 30, 338-377, (1998).

[63] K. E. Gustafson and D. K. M. Rao, Numerical Range: The Field of Values of Linear Operators and Matrices, Springer, New York, 1997.

[64] E. Gutkin and R. Johnson, Intersection theory for linear eigenvalue problems, J. reine angew. Math. 401, 1-24 (1989).

[65] E. Hille, Lectures on Ordinary Differential Equations, Addison-Wesley, Reading, 1969.

[66] D. B. Hinton and A. Schneider, On the Titchmarsh-Weyl coefficients for singular SHermitian Systems I, Math. Nachr. 163, 323-342 (1993).

[67] D. B. Hinton and A. Schneider, On the Titchmarsh-Weyl coefficients for singular SHermitian Systems II, Math. Nachr. 185, 67-84 (1997).

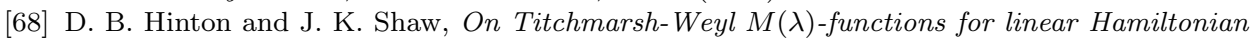
systems, J. Diff. Eqs. 40, 316-342 (1981).

[69] D. B. Hinton and J. K. Shaw, On the spectrum of a singular Hamiltonian system, Quaest. Math. 5, 29-81 (1982).

[70] D. B. Hinton and J. K. Shaw, Hamiltonian systems of limit point or limit circle type with both endpoints singular, J. Diff. Eqs. 50, 444-464 (1983).

[71] D. B. Hinton and J. K. Shaw, On boundary value problems for Hamiltonian systems with two singular points, SIAM J. Math. Anal. 15, 272-286 (1984). 
[72] D. B. Hinton and J. K. Shaw, On the spectrum of a singular Hamiltonian system, II, Quaest. Math. 10, 1-48 (1986).

[73] M. Jodeit and B. M. Levitan, Isospectral vector-valued Sturm-Liouville problems, Lett. Math. Phys. 43, 117-122 (1998).

[74] M. Jodeit and B. M. Levitan, The isospectrality problem for some vector-valued SturmLiouville boundary problems, preprint, 1999.

[75] R. A. Johnson, m-Functions and Floquet exponents for linear differential systems, Ann. Mat. Pura Appl., Ser. 4, 147, 211-248 (1987).

[76] R. Johnson and J. Moser, The rotation number for almost periodic potentials, Commun. Math. Phys. 84, 403-438 (1982).

[77] R. Johnson, S. Novo, and R. Obaya, Ergodic properties and Weyl M-functions for random linear Hamiltonian systems, Proc. Roy. Soc. Edinburgh 130A, 1045-1079 (2000).

[78] V. I. Kogan and F. S. Rofe-Beketov, On square-integrable solutions of symmetric systems of differential equations of arbitrary order, Proc. Roy. Soc. Edinburgh 74A, 1-40 (1974).

[79] S. Kotani, Ljapunov indices determine absolutely continuous spectra of stationary random one-dimensional Schrödinger operators, in Stochastic Analysis, K. Itǒ (ed.), North-Holland, Amsterdam, 1984, pp. 225-247.

[80] S. Kotani, On an inverse problem for random Schrödinger operators, Contemporary Math. 41, 267-281 (1985).

[81] S. Kotani, One-dimensional random Schrödinger operators and Herglotz functions, in Probabilistic Methods in Mathematical Physics, K. Itǒ and N. Ikeda (eds.), Academic Press, New York, 1987, pp. 219-250.

[82] S. Kotani and B. Simon, Stochastic Schrödinger operators and Jacobi matrices on the strip, Commun. Math. Phys. 119, 403-429 (1988).

[83] A. M. Krall, $M(\lambda)$ theory for singular Hamiltonian systems with one singular point, SIAM J. Math. Anal. 20, 664-700 (1989).

[84] A. M. Krall, $M(\lambda)$ theory for singular Hamiltonian systems with two singular points, SIAM J. Math. Anal. 20, 701-715 (1989).

[85] M. G. Krein, Foundations of the theory of $\lambda$-zones of stability of a canonical system of linear differential equations with periodic coefficients, Amer. Math. Soc. Transl. (2) 120, 1-70 (1983).

[86] M. G. Krein, On tests for stable boundedness of solutions of periodic canonical systems, Amer. Math. Soc. Transl. (2) 120, 71-110 (1983).

[87] J. Lacroix, The random Schrödinger operator in a strip, in Probability Measures on Groups VII, H. Heyer (ed.), Lecture Notes in Math. 1064, Springer, Berlin, 1984, pp. 280-297.

[88] J. Lacroix, Computations of the sum of positive Lyapunov exponents for the Llyod model in a strip, in Lyapunov Exponents, L. Arnold and V. Wihstutz (eds.), Lecture Notes in Math. 1186, Springer, Berlin, 1986, pp. 258-264.

[89] A. Laptev and T. Weidl, Sharp Lieb-Thirring inequalities in high dimensions, Acta Math. 184, 87-111 (2000)

[90] Z. L. Leibenzon, The uniqueness of the solution of the inverse problem for ordinary differential operators of order $n \geq 2$ and the transformation of such operators, Sov. Math. Dokl. 3, 100-104 (1962).

[91] Z. L. Leibenzon, A connection between the inverse problem and the completeness of eigenfunctions, Sov. Math. Dokl. 3, 1045-1048 (1962).

[92] M. Lesch and M. Malamud, The inverse spectral problem for first order systems on the half line, Operator Theory: Advances and Applications, Vol. 117, Birkhäuser, Basel, 2000, pp. 199-238.

[93] M. Lesch and M. Malamud, On the number of square integrable solutions and self-adjointness of symmetric first order systems of differential equations, preprint, 2000.

[94] B. M. Levitan and I. S. Sargsjan, Introduction to Spectral Theory, Amer. Math. Soc., Providence, R. I., 1975.

[95] B. M. Levitan and M. Otelbaev, Conditions for self-adjointness of the Schrödinger and Dirac operators, Trans. Moscow. Math. Soc. 42, 139-156 (1982).

[96] B. M. Levitan and I. S. Sargsjan, Sturm-Liouville and Dirac Operators, Kluwer, Dordrecht, 1991.

[97] P. López-Rodriguez, Riesz's theorem for orthogonal matrix polynomials, Constr. Approx. 15, 135-151 (1999). 
[98] F. G. Maksudov, E. M. Bairamov, and R. U. Orudzheva, The inverse scattering problem for an infinite Jacobi matrix with operator elements, Russ. Acad. Sci. Dokl. Math. 45, 366-370 (1992).

[99] M. M. Malamud, Similarity of Volterra operators and related questions of the theory of differential equations of fractional order, Trans. Moscow Math. Soc. 55, 57-122 (1994).

[100] M. M. Malamud, A connection between the potential matrix of the Dirac system and its Wronskian, Dokl. Math. 52, 296-299 (1995).

[101] M. M. Malamud, Uniqueness questions in inverse problems for systems of ordinary differential equations on a finite interval, Trans. Moscow Math. Soc. 60, 173-224 (1999).

[102] M. M. Malamud, Borg type theorems for first-order systems on a finite interval, Funct. Anal. Appl. 33, 64-68 (1999).

[103] Yu. I. Manin, Matrix solitons and bundles over curves with singularities, Funct. Anal. Appl. 12, 286-295 (1978).

[104] V. A. Marchenko, Certain problems in the theory of second-order differential operators, Doklady Akad. Nauk SSSR 72, 457-460 (1950) (Russian).

[105] V. A. Marčenko, Some questions in the theory of one-dimensional linear differential operators of the second order. I, Trudy Moskov. Mat. Obšč. 1, 327-420 (1952) (Russian); English transl. in Amer. Math. Soc. Transl. (2) 101, 1-104 (1973).

[106] V. A. Marchenko, Sturm-Liouville Operators and Applications, Birkhäuser, Basel, 1986.

[107] V. A. Marchenko, Nonlinear Equations and Operator Algebras, Reidel, Dordrecht, 1988.

[108] L. Martinez Alonso and E. Olmedilla, Trace identities in the inverse scattering transform method associated with matrix Schrödinger operators, J. Math. Phys. 23, 2116-2121 (1982).

[109] V. V. Martynov, Condition for discreteness and continuity of the spectrum of a selfadjoint system of first-order differential equations, Sov. Math. Dokl. 6, 1546-1550 (1965).

[110] R. Mennicken, A. L. Sakhnovich, and C. Tretter, Direct and inverse spectral problem for a system of differential equations depending rationally on the spectral parameter, Duke Math. J., to appear.

[111] R. G. Newton and R. Jost, The construction of potentials from the $S$-matrix for systems of differential equations, Nuovo Cim. 1, 590-622 (1955).

[112] E. Olmedilla, L. Martinez Alonso, and F. Guil, Infinite-dimensional Hamiltonian systems associated with matrix Schrödinger operators, Nuovo Cim. 61 B, 49-61 (1981).

[113] S. A. Orlov, Nested matrix disks analytically depending on a parameter, and theorems on the invariance of ranks of radii of limiting disks, Math. USSR Izv. 10, 565-613 (1976).

[114] V. G. Papanicolaou, Trace formulas and the behavior of large eigenvalues, SIAM J. Math. Anal. 26, 218-237 (1995).

[115] V. Q. Phong, The operator equation $A X-X B=C$ with unbounded operators $A$ and $B$ and related abstract Cauchy problems, Math. Z. 208, 567-588 (1991).

[116] F. S. Rofe-Beketov, Expansions in eigenfunctions of infinite systems of differential equations in the non-self-adjoint and self-adjoint cases, Mat. Sb. 51, 293-342 (1960). (Russian).

[117] F. S. Rofe-Beketov, The spectrum of non-selfadjoint differential operators with periodic coefficients, Sov. Math. Dokl. 4, 1563-1566 (1963).

[118] F. S. Rofe-Beketov, Selfadjoint extensions of differential operators in a space of vector functions, Sov. Math. Dokl. 10, 188-192 (1969).

[119] F. S. Rofe-Beketov and A. M. Hol'kin, On the connection between spectral and oscillation properties of the Sturm-Liouville matrix problem, Math. USSR Sbornik 31, 365-378 (1977).

[120] A. L. Sakhnovich, Asymptotic behavior of spectral functions of an $S$-node, Sov. Math. (Iz. VUZ) 32:9, 92-105 (1988).

[121] A. L. Sakhnovich, Nonlinear Schrödinger equation on a semi-axis and an inverse problem associated with it, Ukrain. Math. J. 42, 316-323 (1990).

[122] A. L. Sakhnovich, Spectral functions of a canonical system of order $2 n$, Math. USSR Sbornik 71, 355-369 (1992).

[123] A. Sakhnovich, Iterated Backlund-Darboux transform for canonical systems, J. Funct. Anal. 144, 359-370 (1997).

[124] A. Sakhnovich, Canonical systems and transfer matrix-functions, Proc. Amer. Math. Soc. 125, 1451-1455 (1997).

[125] A. Sakhnovich, Dirac type and canonical systems: spectral functions, direct and inverse problems, preprint, 2001. 
[126] L. A. Sakhnovich, Evolution of spectral data and nonlinear equations, Ukrain. Math. J. 40, 459-461 (1988).

[127] L. A. Sakhnovich, Inverse problems for equations systems, in Matrix and Operator Valued Functions: The Vladimir Petrovich Potapov Memorial Volume, I. Gohberg and L. A. Sakhnovich (eds.), Operator Theory: Advances and Applications, Vol. 72, Birkhäuser, Basel, 1994, pp. 202-211.

[128] L. A. Sakhnovich, Method of operator identities and problems of analysis, St. Petersburg Math. J. 5, 1-69 (1994).

[129] L. A. Sakhnovich, Spectral problem on half-axis, Methods Funct. Anal. Topology 2, 128-140 (1996).

[130] L. A. Sakhnovich, Interpolation Theory and its Applications, Kluwer, Dordrecht, 1997.

[131] L. A. Sakhnovich, Spectral analysis of a class of canonical differential systems, St. Petersburg Math. J. 10, 147-158 (1999).

[132] L. A. Sakhnovich, Spectral Theory of Canonical Differential Systems. Method of Operator Identities, Operator Theory: Advances and Applications, Vol. 107, Birkhäuser, Basel, 1999.

[133] C.-L. Shen and C.-T. Shieh, Two inverse eigenvalue problems for vectorial Sturm-Liouville equations, Inverse Probl. 14, 1331-1343 (1998).

[134] B. Simon, A new aproach to inverse spectral theory, I. Fundamental formalism, Ann. of Math. 150, 1029-1057 (1999).

[135] G. Teschl, Trace formulas and inverse spectral theory for Jacobi operators, Commun. Math. Phys. 196, 175-202 (1998).

[136] G. Teschl, Jacobi Operators and Completely Integrable Nonlinear Lattices, Mathematical Surveys and Monographs, Vol. 72, Amer. Math. Soc., Providence, RI, 2000.

[137] I. Trooshin, Asymptotics for the spectral and Weyl functions of the operator-valued SturmLiouville problem, in "Inverse Problems and Related Topics", G. Nakamura, S. Saitoh, J. K. Seo, and M. Yamamoto (eds.), Chapman \& Hall/CRC, Res. Notes Math. 419, Boca Raton, FL, 2000, pp. 189-208.

[138] M. Wadati and T. Kamijo, On the extension of inverse scattering method, Progr. Theoret. Phys. 52, 397-414 (1974).

[139] J. Weidmann, Oszillationsmethoden für Systeme gewöhnlicher Differentialgleichungen, Math. Z. 119, 349-373 (1971).

[140] J. Weidmann, Spectral Theory of Ordinary Differential Operators, Lecture Notes in Math. 1258, Springer, Berlin, 1987.

[141] W. Weyl, Über gewöhnliche Differentialgleichungen mit Singularitäten und die zugehören Entwicklungen willkürlicher Funktionen, Math. Ann. 68, 220-269 (1910).

[142] V. A. Yakubovich, Nonoscillation of linear periodic Hamiltonian equations, and related topics, St. Petersburg Math. J. 3, 1165-1188 (1992).

[143] V. A. Yakubovich and V. M. Starzhinskii, Linear Differential Equations with Periodic Coefficients 1, Wiley, New York, 1975.

[144] V. A. Yakubovich and V. M. Starzhinskii, Linear Differential Equations with Periodic Coefficients 2, Wiley, New York, 1975.

[145] X. Zhou, Inverse scattering transform for systems with rational spectral dependence, J. Diff. Eqs. 115, 277-303 (1995).

Department of Mathematics, University of Missouri, Columbia, MO 65211, USA

E-mail address: fritz@math.missouri.edu

$U R L:$ http://www.math.missouri.edu/people/fgesztesy.html

Department of Mathematics, University of Missouri, Columbia, MO 65211, USA

E-mail address: makarov@math.missouri.edu

$U R L$ : http://www.math.missouri.edu/people/kmakarov.html

Department of Mathematics, University of Chicago, 5734 South University Avenue, Chicago, IL 60637-1546 USA

E-mail address: kiselev@math.uchicago.edu 